PHYSICAL REVIEW D 96, 063501 (2017)

\title{
Obstructions to Bell CMB experiments
}

\author{
Jérôme Martin* \\ Institut d'Astrophysique de Paris, UMR 7095-CNRS, Université Pierre et Marie Curie, \\ 98 bis boulevard Arago, 75014 Paris, France \\ Vincent Vennin ${ }^{\dagger}$ \\ Laboratoire Astroparticule et Cosmologie, Université Denis Diderot Paris 7, 75013 Paris, France \\ and Institute of Cosmology \& Gravitation, University of Portsmouth, \\ Dennis Sciama Building, Burnaby Road, Portsmouth PO1 3FX, United Kingdom
}

(Received 27 June 2017; published 6 September 2017)

\begin{abstract}
We present a general and systematic study of how a Bell experiment on the cosmic microwave background could be carried out. We introduce different classes of pseudo-spin operators and show that, if the system is placed in a two-mode squeezed state as inflation predicts, they all lead to a violation of the Bell inequality. However, we also discuss the obstacles that one faces in order to realize this program in practice and show that they are probably insurmountable. We suggest alternative methods that could reveal the quantum origin of cosmological structures without relying on Bell experiments.
\end{abstract}

DOI: 10.1103/PhysRevD.96.063501

\section{INTRODUCTION}

According to inflation [1-6], galaxies, clusters of galaxies as well as the cosmic microwave background (CMB) anisotropies [7-12] are of quantum mechanical origin. Conceptually, this fundamental insight is revolutionary since it means that the structures in our Universe are, ultimately, nothing but quantum fluctuations stretched over cosmological distances [13-18] (for reviews, see Refs. [19-25]). Moreover, the quantum state of these fluctuations is a two-mode squeezed state $[26,27]$ which is an entangled state, namely a quantum state that possesses highly non-classical properties. This opens up the possibility to observe genuine quantum effects from the very early Universe in the sky, a fascinating prospect indeed.

However, it has been suggested, maybe surprisingly given the nonclassical character of the CMB quantum state, that achieving this goal may be difficult. The reason is that the quantum-mechanical phase space of cosmological perturbations is made of two noncommuting conjugated variables, the so-called growing and decaying modes. During inflation, the decaying mode decays exponentially. This is why it is, a priori, impossible to measure any quantity related to its amplitude, hence to its commutator with the growing mode. Detecting quantum correlations via this commutator therefore seems intractable in practice. For instance, its has been shown $[28,29]$ that the two-point quantum correlation functions involving the growing mode only, i.e. the ones that are realistically observable, are, in practice, indistinguishable from their "classical" counterparts.

\footnotetext{
*jmartin@iap.fr

vennin@apc.univ-paris7.fr
}

Nevertheless, the question of designing Bell CMB experiments [30-35] is worth investigating for the three following reasons. First, the qualitative argument mentioned above is rather vague and it is important to study how it manifests itself in a concrete and explicit attempt to carry out a Bell experiment on the CMB. Only then can one assess how severe it is and whether it can be circumvented or not. Second, the argument holds for measurements of correlation functions at a single wave number $\boldsymbol{k}$, while Bell CMB experiments typically involve correlators between quantities calculated at modes $\boldsymbol{k}$ and $\boldsymbol{- k}$, which makes the argument incomplete. We will see that indeed, Bell inequalities can be violated even in the limit where the decaying mode vanishes. Third, there are nonminimal cases where the decaying mode may be accessible, either because it does not vanish on large scales during inflation if sourced by isocurvature perturbations [36] for instance, or if, as proposed in Ref. [31], it couples to other fields and becomes observable through them. In such cases, it remains to determine how Bell inequality violations could be extracted from the CMB data.

This is why in this article, we carry out a systematic study and discussion of all the obstacles one faces when trying to implement a Bell CMB experiment. This leads us to considerations, for instance, the use of CMB pseudospin operators, or the connection between the positivity of the Wigner function and the so-called proper variables, that shed new light on these issues. Based on the results obtained here, we also discuss alternative methods that may reveal the quantum origin of cosmological perturbations without relying on Bell experiments.

The paper is organized as follows. In the next section, Sec. II, we briefly review the theory of inflationary cosmological perturbations of quantum-mechanical origin 
and discuss the motivations of the present study. Then, in Sec. III, we explain how a cosmic Bell experiment could be designed. In a first step, see Sec. III A, we briefly review how the Bell experiment is usually carried out in a standard context. Then, we proceed by analogy. In Sec. III B, we explain how a dichotomic variable can be extracted out from a continuous variable system, introduce a first class of pseudo-spin operators and show that they lead to a violation of the Bell inequality. In Sec. IIIC and Sec. III D, we consider two other sets of possible pseudo-spin operators also leading to a Bell inequality violation. In Sec. IV, we discuss and interpret these results. In Sec. IVA, we demonstrate that our results are consistent with a known theorem stating that, if the Wigner function of the system is positive (which is the case in cosmology), then a Bell inequality violation can occur only for improper dynamical variables. In Sec. IV B, we study whether the pseudo-spin variables can be measured experimentally and conclude that the answer is probably negative. In Sec. IV C, we study the robustness of our results to decoherence effects. Finally, in Sec. V, we present our conclusions. The last section of the paper is Appendix. A where we detail the calculation of the correlation function of one of the $\hat{\mathcal{S}}_{x}$ operator introduced in this article.

\section{MOTIVATION}

Inflation produces two types of fluctuations, scalars and tensors. In the following, we restrict ourselves to scalar perturbations since they are the only ones observed so far and they decouple from the tensor ones. However, this does not limit in any way the generality of our argument since tensor modes could be studied in a similar fashion. The evolution of scalar perturbations is controlled by the following Hamiltonian [13,19]

$$
\begin{aligned}
\hat{H}= & \int_{\mathbb{R}^{3}} \mathrm{~d}^{3} \boldsymbol{k}\left[\frac{k}{2}\left(\hat{c}_{\boldsymbol{k}} \hat{c}_{\boldsymbol{k}}^{\dagger}+\hat{c}_{-\boldsymbol{k}} \hat{c}_{-\boldsymbol{k}}^{\dagger}\right)\right. \\
& \left.-\frac{i}{2} \frac{z^{\prime}}{z}\left(\hat{c}_{\boldsymbol{k}} \hat{c}_{-\boldsymbol{k}}-\hat{c}_{-\boldsymbol{k}}^{\dagger} \hat{c}_{\boldsymbol{k}}^{\dagger}\right)\right],
\end{aligned}
$$

where $\hat{c}_{\boldsymbol{k}}$ and $\hat{c}_{\boldsymbol{k}}^{\dagger}$ are respectively the creation and annihilation operators satisfying the standard commutation relation $\left[\hat{c}_{\boldsymbol{k}}, \hat{c}_{p}^{\dagger}\right]=\delta(\boldsymbol{k}-\boldsymbol{p})$. In the above expression, the quantity $z$ is defined to be $z \equiv a M_{\mathrm{PI}} \sqrt{2 \epsilon_{1}}$, where $a(t)$ is the Friedmann-Lemaitre-Robertson-Walker (FLRW) scale factor, $M_{\mathrm{Pl}}$ the reduced Planck mass and $\epsilon_{1}$ the first Hubble flow parameter, $\epsilon_{1} \equiv-\dot{H} / H^{2}, H=\dot{a} / a$ being the Hubble parameter. A dot denotes a derivative with respect to cosmic time $t$ while a prime represents a derivative with respect to conformal time $\eta$ with $\mathrm{d} t=a \mathrm{~d} \eta$. The creation and annihilation operators are related to the scalar curvature perturbations $\hat{\zeta}_{k}$ through $\hat{v}_{k}=\left(\hat{c}_{k}+\hat{c}_{-k}^{\dagger}\right) / \sqrt{2 k}$ and $\hat{p}_{k}=$ $-i \sqrt{k / 2}\left(\hat{c}_{k}-\hat{c}_{-k}^{\dagger}\right)$ by $\hat{\zeta}_{k}=\hat{v}_{k} / z$ and $\hat{\zeta}_{k}^{\prime}=\hat{p}_{k} / z$. The quantity $\hat{v}_{\boldsymbol{k}}$ is the so-called Mukhanov-Sasaki variable. We also introduce the quantities $\hat{q}_{k}$ and $\hat{\pi}_{k}$ given by $\hat{q}_{k}=$ $\left(\hat{c}_{k}+\hat{c}_{k}^{\dagger}\right) / \sqrt{2 k}$ and $\hat{\pi}_{k}=-i \sqrt{k / 2}\left(\hat{c}_{k}-\hat{c}_{k}^{\dagger}\right)$. The advantage of these variables is that they are defined for a fixed wave number $\boldsymbol{k}$ (while $\zeta_{\boldsymbol{k}}$ mixes $\boldsymbol{k}$ and $\boldsymbol{- k}$ ) and they are Hermitian. In this sense, they really play the role of the position and momentum at the scale $\boldsymbol{k}$. The relation between these two sets of variables is easily obtained, see for instance Eqs. (51) and (52) of Ref. [29], and is given by

$$
\begin{gathered}
\hat{v}_{\boldsymbol{k}}=\frac{1}{2}\left[\hat{q}_{\boldsymbol{k}}+\hat{q}_{-\boldsymbol{k}}+\frac{i}{k}\left(\hat{\pi}_{\boldsymbol{k}}-\hat{\pi}_{-\boldsymbol{k}}\right)\right], \\
\hat{p}_{\boldsymbol{k}}=\frac{1}{2}\left[k\left(\hat{q}_{\boldsymbol{k}}-\hat{q}_{-\boldsymbol{k}}\right)+i\left(\hat{\pi}_{\boldsymbol{k}}+\hat{\pi}_{-k}\right)\right] .
\end{gathered}
$$

The Hamiltonian given by Eq. (1) is well known and represents a collection of parametric oscillators. The above discussion shows that the full Hilbert space of the system $\mathcal{E}$ can be factorized into independent products of Hilbert spaces for modes $\boldsymbol{k}$ and $\boldsymbol{- k}$,

$$
\mathcal{E}=\Pi_{k \in \mathbb{R}^{3+}} \mathcal{E}_{\boldsymbol{k}} \otimes \mathcal{E}_{-\boldsymbol{k}} .
$$

In other words, the CMB fluctuations can be viewed as a product of independent bipartite systems. If, initially, the quantum state is the vacuum state $\left|0_{k}, 0_{-k}\right\rangle$, then, under the Hamiltonian (1), it evolves into a two-mode squeezed state $[21,22,26-28]$

$$
\left|\Psi_{2 \mathrm{sq}}\right\rangle=\frac{1}{\cosh r_{k}} \sum_{n=0}^{\infty} \mathrm{e}^{-2 i n \varphi_{k}} \tanh ^{n} r_{k}\left|n_{k}, n_{-k}\right\rangle,
$$

where $\left|n_{k}\right\rangle$ is an eigenvector of the particle number operator, $N_{k}=\hat{c}_{k}^{\dagger} \hat{c}_{k}$. In the above expression, $r_{k}$ and $\varphi_{k}$ are respectively the squeezing parameter and squeezing angle. They are functions of time that depend on the details of the inflationary background, but are such that on subHubble scales, i.e. when $k \gg a H, r_{k} \rightarrow 0$ and $\varphi_{k} \rightarrow-\pi / 4$, while on super-Hubble scales, i.e. when $k \ll a H, r_{k} \rightarrow \infty$ and $\varphi \rightarrow-\pi / 2{ }^{1}$ More precisely, on super-Hubble scales,

${ }^{1}$ In Ref. [29], the two-mode squeezed state was written

$$
\left|\Psi_{2 \mathrm{sq}}\right\rangle=\frac{1}{\cosh r_{k}} \sum_{n=0}^{\infty} \mathrm{e}^{2 i n \varphi_{k}}(-1)^{n} \tanh ^{n} r_{k}\left|n_{k}, n_{-k}\right\rangle,
$$

which implies a different definition of the squeezing angle, namely $\varphi_{k} \rightarrow-\left(\varphi_{k}+\pi / 2\right)$. With the convention of Ref. [29], the squeezing angles goes to zero on large scales. Moreover in Ref. [32], the symmetries of the problem allows the analysis to be restricted to the range $\varphi_{k} \in[0, \pi / 4]$. During inflation, $\varphi_{k} \in$ $[-\pi / 2,-\pi / 4]$ and if one wants to use the results of Ref. [32], this can be done by changing $\varphi_{k} \rightarrow \varphi_{k}+\pi / 2$. 
$r_{k}$ is of order the number of $e$-folds $N \equiv \ln a$ spent outside the Hubble radius, which is $\sim 50$ for the modes probed in the CMB.

The classical limit in quantum mechanics is subtle and this question is perfectly illustrated in the case of a twomode squeezed state. Usually, this state is considered as the prototype of a "nonclassical" quantum state. In this language, a "classical" state would typically be a coherent state, namely a state whose Wigner function follows the trajectory of the classical system in phase space with minimum dispersion. On the contrary, the Wigner function of a squeezed state has a large extension in phase space (to be more precise in a specific direction). Moreover, the extension is all the greater when the squeezing parameter is large. And this is precisely the case in cosmology where the values of $r_{k}$ are much larger than what can be achieved in the laboratory with conventional systems. In this sense, the sky is placed in a highly "quantum-mechanical" state.

On the other hand, a system is also said to be classical if its Wigner function is positive. Indeed, it is well known that interference terms, which are typical signatures of a quantum behavior, cause oscillations in the Wigner function and make it negative for some values of position and momentum. But a squeezed state is a Gaussian state and, therefore, its Wigner function is always positive. In this sense, and contrary to the previous criterion, the system is classical.

However, as is apparent in Eq. (5), the two-mode squeezed state is also an entangled state. Clearly, entanglement is a genuine quantum-mechanical feature and, therefore, this feature brings us back to the first conclusion. Moreover, the larger $r_{k}$, the larger the entanglement as confirmed by a calculation of the quantum discord ${ }^{2}[37,38]$ (for a review see Ref. [39]) performed in Ref. [29]

$$
\begin{aligned}
\delta(\boldsymbol{k},-\boldsymbol{k})= & \cosh ^{2} r_{k} \log _{2}\left(\cosh ^{2} r_{k}\right) \\
& -\sinh ^{2} r_{k} \log _{2}\left(\sinh ^{2} r_{k}\right) \\
\simeq & \frac{2}{\ln 2} r_{k}-2+\frac{1}{\ln 2}+\mathcal{O}\left(\mathrm{e}^{-2 r_{k}}\right),
\end{aligned}
$$

where the last expansion is valid for large values of the squeezing parameter.

If the CMB does not have a classical behavior, this means that, at least in principle, one should be able to design experiments allowing us to exhibit its nonclassical properties. One usually characterizes the $\mathrm{CMB}$ by the correlation functions of curvature perturbations. If the system is really "non classical," then these correlation functions should differ according to whether one calculates them with a two-mode squeezed state or with a classical state. Here, by classical state, we mean a state which

\footnotetext{
${ }^{2}$ For pure states such as Eq. (5), the quantum discord becomes a measure of quantum entanglement. More specifically in that case, it equals the entropy of entanglement.
}

contains classical correlations only and has zero discord [40]. In Ref. [29], it has been shown that such a state can be chosen so as to exactly reproduce the two-point correlation function $\langle\hat{\zeta}(\eta, x) \hat{\zeta}(\eta, \boldsymbol{y})\rangle$. But, in accordance with the fact that the discord of the system is large, this would then imply correlation functions $\left\langle\hat{\zeta}(\eta, \boldsymbol{x}) \hat{\zeta}^{\prime}(\eta, \boldsymbol{y})+\hat{\zeta}^{\prime}(\eta, \boldsymbol{x}) \hat{\zeta}(\eta, \boldsymbol{y})\right\rangle$ and/or $\left\langle\hat{\zeta}^{\prime}(\eta, \boldsymbol{x}) \hat{\zeta}^{\prime}(\eta, \boldsymbol{y})\right\rangle$ that strongly differ from their twomode squeezed state counterparts. At this stage, one would therefore be tempted to claim that, indeed, quantum signatures can be detected in the sky. However, in practice, the quantity $\hat{\zeta}^{\prime}(\eta, \boldsymbol{x})$ is not observable since this is the decaying mode. Importantly at the conceptual level, we see that the argument is not a theoretical argument but a practical one. In principle, the difference between twopoint correlation functions calculated in a classical or quantum-mechanical contexts exist and are observable but, in practice, due to the smallness of the decaying mode amplitude, this is hidden to us, probably forever.

If one considers higher correlation functions however (such as the four-point correlation function and higher), even when relying on the growing mode only, there is no classical state that can reproduce the predictions of the twomode squeezed state [29]. This is consistent with the theorem [41-43] stating that the only classical Gaussian states are product states, i.e. uncorrelated states. Since the CMB statistics is constrained to be Gaussian or almost Gaussian [12], and since the bipartite systems of Eq. (4) are necessarily correlated [if not one can simply perform a phase space transformation similar to Eqs. (47) and (48) of Ref. [29] to obtain a correlated system], these correlations must therefore possess nonvanishing discord. For a pure state, nonvanishing discord is equivalent to entanglement and this implies that Bell inequalities can (at least in principle) be violated. This is the possibility we investigate in this work. Otherwise, this implies that the CMB is placed in a mixed, decohered state, another interesting possibility that we will discuss in a separate article, see also Sec. IV C.

\section{BELL CMB EXPERIMENT WITH PSEUDO-SPIN OPERATORS}

\section{A. Bell inequality with spins}

In order to see how we could design a Bell CMB experiment, let us first, very briefly, recall how this is done in a conventional situation, the so-called Clauser, Horne, Shimony, and Holt (CHSH) setup [44]. The idea is to consider a bipartite system whose Hilbert space is written as $\mathcal{H}=\mathcal{H}_{A} \otimes \mathcal{H}_{B}$. Typically, $A$ and $B$ are two particles whose spin along the $z$ direction are correlated, see Fig. 1. The state of the system is assumed to be

$$
|\Psi\rangle=\frac{1}{\sqrt{2}}(|+,-\rangle-|-,+\rangle),
$$

where $| \pm\rangle$ are eigenstates of $\hat{S}_{z}$ with, respectively, eigenvalues \pm 1 . The spin of system $A$ is measured along the 


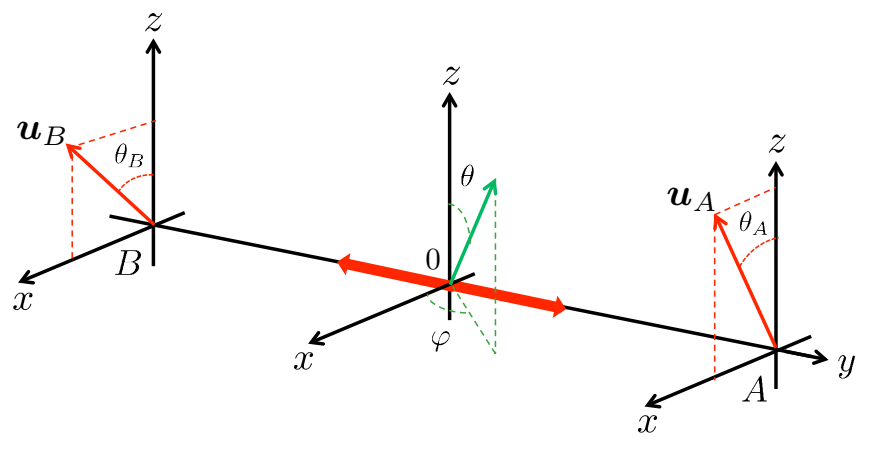

FIG. 1. Standard setup for Bell experiment. Two spin particles are emitted at the origin 0 and travel in opposite directions. Their spin is then measured at $A$ and $B$ along the directions $\boldsymbol{u}_{A}$ and $\boldsymbol{u}_{B}$.

direction characterized by the angle $\theta_{A}$ in the $(x, z)$ plane, namely along the vector $\boldsymbol{u}_{A}$ and the spin of system $B$ is measured along the direction $\boldsymbol{u}_{B}$, see Fig. 1. In general, if $\boldsymbol{S} \cdot \boldsymbol{u}$ is the spin operator along the direction $\boldsymbol{u}=(\sin \theta \cos \varphi, \sin \theta \sin \varphi, \cos \theta)$, the eigenstates of $\boldsymbol{S} \cdot \boldsymbol{u}$ are $\left|{ }_{\boldsymbol{u}}\right\rangle=\cos (\theta / 2) e^{-i \varphi / 2}|+\rangle+\sin (\theta / 2) e^{i \varphi / 2}|-\rangle$ and $\left|-_{\boldsymbol{u}}\right\rangle=-\sin (\theta / 2) e^{-i \varphi / 2}|+\rangle+\cos (\theta / 2) e^{i \varphi / 2}|-\rangle$ with eigenvalues \pm 1 . Then, one can introduce the Bell operator

$$
\begin{aligned}
\hat{\mathcal{B}}_{\mathrm{CHSH}}(A, B)= & \boldsymbol{u}_{A} \cdot \hat{\boldsymbol{S}}_{A} \otimes \boldsymbol{u}_{B} \cdot \hat{\boldsymbol{S}}_{B}+\boldsymbol{u}_{A} \cdot \hat{\boldsymbol{S}}_{A} \otimes \boldsymbol{u}_{B}^{\prime} \cdot \hat{\boldsymbol{S}}_{B} \\
& +\boldsymbol{u}_{A}^{\prime} \cdot \hat{\boldsymbol{S}}_{A} \otimes \boldsymbol{u}_{B} \cdot \hat{\boldsymbol{S}}_{B}-\boldsymbol{u}_{A}^{\prime} \cdot \hat{\boldsymbol{S}}_{A} \otimes \boldsymbol{u}_{B}^{\prime} \cdot \hat{\boldsymbol{S}}_{B},
\end{aligned}
$$

where $\boldsymbol{u}_{A}, \boldsymbol{u}_{A}^{\prime}, \boldsymbol{u}_{B}$, and $\boldsymbol{u}_{B}^{\prime}$ are four different vectors, all located in the $(x, z)$ plane (and, therefore, with vanishing azimuthal angles). Then, one has to calculate the mean value of the Bell operator in the state (8). One can show that $\left\langle\hat{\mathcal{B}}_{\mathrm{CHSH}}(A, B)\right\rangle=E\left(\theta_{A}, \theta_{B}\right)+E\left(\theta_{A}, \theta_{B}^{\prime}\right)+E\left(\theta_{A}^{\prime}, \theta_{B}\right)-$ $E\left(\theta_{A}^{\prime}, \theta_{B}^{\prime}\right) \quad$ with $\quad E\left(\theta_{A}, \theta_{B}\right) \equiv\left\langle\boldsymbol{u}_{A} \cdot \hat{\boldsymbol{S}}_{A} \otimes \boldsymbol{u}_{B} \cdot \hat{\boldsymbol{S}}_{B}\right\rangle=$ $-\cos \left(\theta_{A}-\theta_{B}\right)$. If, for instance, one chooses $\theta_{A}-\theta_{B}=$ $\pi / 4, \theta_{A}-\theta_{B}^{\prime}=\theta_{A}^{\prime}-\theta_{B}=-\pi / 4$ and $\theta_{A}^{\prime}-\theta_{B}^{\prime}=-3 \pi / 4$, then $\left\langle\hat{\mathcal{B}}_{\mathrm{CHSH}}(A, B)\right\rangle=-2 \sqrt{2}$. Since $\mid\left\langle\hat{\mathcal{B}}_{\mathrm{CHSH}}(A, B)\right|>2$, the Bell inequality is violated and this cannot be accounted for in a theory with local realism. As is well known, this has been experimentally confirmed [45-48]. Very recently, this has even been observed with a setup where the detectors are controlled by the light coming from distant stars [49].

Our goal is now to design a similar approach but with the CMB.

\section{B. Banaszek-Wodkiewicz (BW) spin operators}

The first difficulty that we meet is that we deal with a continuous variable system. Indeed $\hat{\zeta}_{\boldsymbol{k}}$ (or the MukhanovSasaki variable $\hat{v}_{k}$ ) are continuous complex operators and have a continuous spectrum, not a discrete one with two eigenvalues \pm 1 . However, for any continuous variable system, it is possible to introduce fictitious or pseudo-spin operators. They have been discussed by Banaszek and Wodkiewics (BM) in Ref. [50] and Chen, Pan, Hou, and Zhang in Ref. [51] and are defined by [50-53]

$$
\begin{aligned}
& \hat{s}_{x}(\boldsymbol{k})=\sum_{n=0}^{\infty}\left(\left|2 n_{k}+1\right\rangle\left\langle 2 n_{k}|+| 2 n_{k}\right\rangle\left\langle 2 n_{k}+1\right|\right) \\
& \hat{s}_{y}(\boldsymbol{k})=i \sum_{n=0}^{\infty}\left(\left|2 n_{k}\right\rangle\left\langle 2 n_{k}+1|-| 2 n_{k}+1\right\rangle\left\langle 2 n_{k}\right|\right) \\
& \hat{s}_{z}(\boldsymbol{k})=\sum_{n=0}^{\infty}\left(\left|2 n_{k}+1\right\rangle\left\langle 2 n_{k}+1|-| 2 n_{k}\right\rangle\left\langle 2 n_{k}\right|\right),
\end{aligned}
$$

and similar expression for the mode $-\boldsymbol{k}$. The states $\left|n_{\boldsymbol{k}}\right\rangle$ are the eigenvectors of the particle number operator already introduced before. It is easy to verify that these operators satisfy the usual $S U(2)$ commutation relations for a spin, namely $\left[\hat{s}_{x}, \hat{s}_{y}\right]=2 i \hat{s}_{z},\left[\hat{s}_{x}, \hat{s}_{z}\right]=-2 i \hat{s}_{y}$ and $\left[\hat{s}_{y}, \hat{s}_{z}\right]=2 i \hat{s}_{x}$. Moreover, if one defines a fictitious unit vector $\boldsymbol{n}=\left(\sin \theta_{n} \cos \varphi_{n}, \sin \theta_{n} \sin \varphi_{n}, \cos \theta_{n}\right)$, then one has $(\boldsymbol{n} \cdot \hat{\boldsymbol{s}})^{2}=\hat{\mathrm{I}}$ which means that the outcome of a measurement of the Hermitian operator $\boldsymbol{n} \cdot \boldsymbol{s}$ is, as expected, \pm 1 . Therefore, we have achieved a first goal, namely define a dichotomic variable from a continuous variable system. From this point, one can then proceed by analogy. We can indeed define the Bell operator by

$$
\begin{aligned}
\hat{\mathcal{B}}_{\mathrm{BW}}(\boldsymbol{k},-\boldsymbol{k})= & \boldsymbol{n} \cdot \hat{\boldsymbol{s}}(\boldsymbol{k}) \otimes \boldsymbol{m} \cdot \hat{\boldsymbol{s}}(-\boldsymbol{k}) \\
& +\boldsymbol{n} \cdot \hat{\boldsymbol{s}}(\boldsymbol{k}) \otimes \boldsymbol{m}^{\prime} \cdot \hat{\boldsymbol{s}}(-\boldsymbol{k}) \\
& +\boldsymbol{n}^{\prime} \cdot \hat{\boldsymbol{s}}(\boldsymbol{k}) \otimes \boldsymbol{m} \cdot \hat{\boldsymbol{s}}(-\boldsymbol{k}) \\
& -\boldsymbol{n}^{\prime} \cdot \hat{\boldsymbol{s}}(\boldsymbol{k}) \otimes \boldsymbol{m}^{\prime} \cdot \hat{\boldsymbol{s}}(-\boldsymbol{k}),
\end{aligned}
$$

where $\boldsymbol{n}, \boldsymbol{n}^{\prime}, \boldsymbol{m}$, and $\boldsymbol{m}^{\prime}$ are four unit vectors, since this mimics exactly Eq. (9). Then, one has to calculate the mean value of this operator, not in a state similar to the one given in Eq. (8) though but, of course, in a two-mode squeezed state as Eq. (5). This gives

$$
\begin{aligned}
\left\langle\Psi_{2 \mathrm{sq}}\left|\hat{\mathcal{B}}_{\mathrm{BW}}(\boldsymbol{k},-\boldsymbol{k})\right| \Psi_{2 \mathrm{sq}}\right\rangle= & E\left(\theta_{n}, \theta_{m}\right)+E\left(\theta_{n}, \theta_{m^{\prime}}\right) \\
& +E\left(\theta_{n^{\prime}}, \theta_{m}\right)-E\left(\theta_{n^{\prime}}, \theta_{m^{\prime}}\right),
\end{aligned}
$$

where the correlation function $E(\boldsymbol{n}, \boldsymbol{m})$ is defined by

$$
E(\boldsymbol{n}, \boldsymbol{m})=\left\langle\Psi_{2 \mathrm{sq}}|\boldsymbol{n} \cdot \hat{\boldsymbol{s}}(\boldsymbol{k}) \otimes \boldsymbol{m} \cdot \hat{\boldsymbol{s}}(-\boldsymbol{k})| \Psi_{2 \mathrm{sq}}\right\rangle .
$$

If we choose all azimuthal angles to be zero (as it is the case in the standard setup, see the previous subsection), then one has $\boldsymbol{n} \cdot \hat{\boldsymbol{s}}=\sin \theta_{n} \hat{\boldsymbol{s}}_{x}+\cos \theta_{n} \hat{\boldsymbol{s}}_{z}$ and a straightforward calculation shows that $\left\langle\Psi_{2 \mathrm{sq}}\left|\hat{\boldsymbol{s}}_{x}(\boldsymbol{k}) \otimes \hat{\boldsymbol{s}}_{z}(-\boldsymbol{k})\right| \Psi_{2 \mathrm{sq}}\right\rangle=0$. As a consequence, one can write 


$$
E\left(\theta_{n}, \theta_{m}\right)=\left\langle\Psi_{2 \mathrm{sq}}\left|\hat{s}_{z}(\boldsymbol{k}) \otimes \hat{s}_{z}(-\boldsymbol{k})\right| \Psi_{2 \mathrm{sq}}\right\rangle \cos \theta_{n} \cos \theta_{m}+\left\langle\Psi_{2 \mathrm{sq}}\left|\hat{s}_{x}(\boldsymbol{k}) \otimes \hat{s}_{x}(-\boldsymbol{k})\right| \Psi_{2 \mathrm{sq}}\right\rangle \sin \theta_{n} \sin \theta_{m} .
$$

Finally, choosing (for instance) the configuration $\theta_{n}=0, \theta_{n^{\prime}}=\pi / 2$ and $\theta_{m^{\prime}}=-\theta_{m}$ leads to the following expression

$$
\left\langle\Psi_{2 \mathrm{sq}}\left|\hat{\mathcal{B}}_{\mathrm{BW}}(\boldsymbol{k},-\boldsymbol{k})\right| \Psi_{2 \mathrm{sq}}\right\rangle=2\left[\cos \theta_{m}\left\langle\Psi_{2 \mathrm{sq}}\left|\hat{s}_{z}(\boldsymbol{k}) \otimes \hat{s}_{z}(-\boldsymbol{k})\right| \Psi_{2 \mathrm{sq}}\right\rangle+\sin \theta_{m}\left\langle\Psi_{2 \mathrm{sq}}\left|\hat{s}_{x}(\boldsymbol{k}) \otimes \hat{s}_{x}(-\boldsymbol{k})\right| \Psi_{2 \mathrm{sq}}\right\rangle\right]
$$

One can then optimize the choice of $\theta_{m}$ in order to obtain the largest value of $\left\langle\Psi_{2 \mathrm{sq}}\left|\hat{\mathcal{B}}_{\mathrm{BW}}(\boldsymbol{k},-\boldsymbol{k})\right| \Psi_{2 \mathrm{sq}}\right\rangle$. This leads to $\theta_{m}^{\mathrm{opt}}=\arctan \left[\left\langle\Psi_{2 \mathrm{sq}}\left|\hat{s}_{x}(\boldsymbol{k}) \otimes \hat{s}_{x}(-\boldsymbol{k})\right| \Psi_{2 \mathrm{sq}}\right\rangle /\left\langle\Psi_{2 \mathrm{sq}}\left|\hat{s}_{z}(\boldsymbol{k}) \otimes \hat{s}_{z}(-\boldsymbol{k})\right| \Psi_{2 \mathrm{sq}}\right\rangle\right]$ and, therefore, for this optimal configuration,

$$
\left\langle\Psi_{2 \mathrm{sq}}\left|\hat{\mathcal{B}}_{\mathrm{BW}}(\boldsymbol{k},-\boldsymbol{k})\right| \Psi_{2 \mathrm{sq}}\right\rangle=2 \sqrt{\left\langle\Psi_{2 \mathrm{sq}}\left|\hat{s}_{z}(\boldsymbol{k}) \otimes \hat{s}_{z}(-\boldsymbol{k})\right| \Psi_{2 \mathrm{sq}}\right\rangle^{2}+\left\langle\Psi_{2 \mathrm{sq}}\left|\hat{s}_{x}(\boldsymbol{k}) \otimes \hat{s}_{x}(-\boldsymbol{k})\right| \Psi_{2 \mathrm{sq}}\right\rangle^{2}}
$$

For the two-mode squeezed state (5), one can show that $\left\langle\Psi_{2 \mathrm{sq}}\left|\hat{s}_{z}(\boldsymbol{k}) \otimes \hat{s}_{z}(-\boldsymbol{k})\right| \Psi_{2 \mathrm{sq}}\right\rangle=1$. Already at this stage, one sees that the Bell inequality is violated as soon as $\left\langle\Psi_{2 \mathrm{sq}}\left|\hat{s}_{x}(\boldsymbol{k}) \otimes \hat{s}_{x}(-\boldsymbol{k})\right| \Psi_{2 \mathrm{sq}}\right\rangle \neq 0$. From the state (5), one has

$$
\left\langle\Psi_{2 \mathrm{sq}}\left|\hat{s}_{x}(\boldsymbol{k}) \otimes \hat{s}_{x}(-\boldsymbol{k})\right| \Psi_{2 \mathrm{sq}}\right\rangle=\tanh \left(2 r_{k}\right) \cos \left(2 \varphi_{k}\right) .
$$

Notice that, in the case of a vanishing squeezing angle, this result was already derived in Ref. [53] but in a different context. The case $\varphi_{k} \neq 0$ is new. The previous expression clearly demonstrates that the Bell inequality is violated for any nonvanishing value of $r_{k}$ if $\varphi_{k} \neq \pi / 4 \pm \pi / 2$ (with this configuration) as can be seen in the left panel of Fig. 2. So we have the confirmation that, at least at this level of the analysis, the $\mathrm{CMB}$ is an interesting playground to observe a cosmic violation of Bell inequality. Moreover, on superHubble scales, one has $r_{k} \rightarrow \infty$ and $\varphi_{k} \rightarrow-\pi / 2$. In this limit one has $\left\langle\Psi_{2 \mathrm{sq}}\left|\hat{\mathcal{B}}_{\mathrm{BW}}(\boldsymbol{k},-\boldsymbol{k})\right| \Psi_{2 \mathrm{sq}}\right\rangle \rightarrow 2 \sqrt{2}$. This number is the so-called Cirel'son bound [54] and represents the maximal value that the Bell operator can take in quantum mechanics. We therefore conclude that the CMB is placed in a quantum state that maximally violates the Bell inequality. In fact, this result is not so surprising since we have seen that the CMB quantum state has a very large quantum discord. It can even be shown that, in the limit $r_{k} \rightarrow+\infty$, the two-mode squeezed state tends towards the Einstein-Podolski-Rosen (EPR) state [55].

\section{Gour-Khanna-Mann-Revzen (GKMR) spin operators}

Given the result of the previous subsection, a natural question is whether the choice of the pseudospin operators is unique. In fact it is not. An alternative set of pseudo-spin operators has also been considered By Gour, Khanna, Mann, and Revzen in Refs. $[53,56]$ and it is interesting to discuss how they can be used in a cosmological context and, of course, if they lead to a Bell inequality violation. Let us first introduce $\left|\mathcal{E}_{k}\right\rangle$ and $\left|\mathcal{O}_{k}\right\rangle$ by

$$
\begin{aligned}
& \left|\mathcal{E}_{k}\right\rangle=\frac{1}{\sqrt{2}}\left(\left|q_{k}\right\rangle+\left|-q_{k}\right\rangle\right), \\
& \left|\mathcal{O}_{k}\right\rangle=\frac{1}{\sqrt{2}}\left(\left|q_{k}\right\rangle-\left|-q_{k}\right\rangle\right),
\end{aligned}
$$

where we recall that $\hat{q}_{k}$ is the operator playing the role of position in the subspace $\mathcal{E}_{\boldsymbol{k}}$ (recall that the operator $\hat{v}_{\boldsymbol{k}}$ mixes the modes $\boldsymbol{k}$ and $-\boldsymbol{k}$ ). Then, one can define the following operators

$$
\begin{aligned}
& \hat{\mathcal{S}}_{x}=\int_{0}^{+\infty} \mathrm{d} q_{\boldsymbol{k}}\left(\left|\mathcal{E}_{\boldsymbol{k}}\right\rangle\left\langle\mathcal{O}_{\boldsymbol{k}}|+| \mathcal{O}_{\boldsymbol{k}}\right\rangle\left\langle\mathcal{E}_{\boldsymbol{k}}\right|\right), \\
& \hat{\mathcal{S}}_{y}=i \int_{0}^{+\infty} \mathrm{d} q_{\boldsymbol{k}}\left(\left|\mathcal{O}_{\boldsymbol{k}}\right\rangle\left\langle\mathcal{E}_{\boldsymbol{k}}|-| \mathcal{E}_{\boldsymbol{k}}\right\rangle\left\langle\mathcal{O}_{\boldsymbol{k}}\right|\right), \\
& \hat{\mathcal{S}}_{z}=-\int_{0}^{+\infty} \mathrm{d} q_{\boldsymbol{k}}\left(\left|\mathcal{E}_{\boldsymbol{k}}\right\rangle\left\langle\mathcal{E}_{\boldsymbol{k}}|-| \mathcal{O}_{\boldsymbol{k}}\right\rangle\left\langle\mathcal{O}_{\boldsymbol{k}}\right|\right),
\end{aligned}
$$

and it is easy to see that, as the notations suggest, they satisfy all the properties required to be the three components of a spin. In fact, one can show that the operator of Eq. (24), which can also be written as $\hat{\mathcal{S}}_{z}=-\int_{-\infty}^{\infty} \mathrm{d} q_{k}\left|q_{k}\right\rangle\left\langle-q_{k}\right|$, is in fact equal to that of Eq. (12), $\hat{s}_{z}=\hat{\mathcal{S}}_{z}$. Indeed, it is straightforward to show that the matrix element $\left\langle m\left|\hat{s}_{z}\right| m^{\prime}\right\rangle$, where $\hat{s}_{z}$ is given by Eq. (12), is equal to $\pm \delta_{m m^{\prime}}$ with a plus sign if $m$ is odd and a minus sign if $m$ is even. On the other hand, for the operator (24), one has

$$
\begin{aligned}
\left\langle m\left|\hat{\mathcal{S}}_{z}\right| m^{\prime}\right\rangle & =-\int_{-\infty}^{+\infty} \mathrm{d} q_{\boldsymbol{k}}\left\langle m \mid q_{\boldsymbol{k}}\right\rangle\left\langle-q_{\boldsymbol{k}} \mid m^{\prime}\right\rangle \\
& =\frac{-(-1)^{m^{\prime}}}{\sqrt{\pi 2^{m+m^{\prime}} m ! m^{\prime} !}} \int_{-\infty}^{+\infty} \mathrm{d} q_{\boldsymbol{k}} H_{m}\left(q_{\boldsymbol{k}}\right) H_{m^{\prime}}\left(q_{\boldsymbol{k}}\right) e^{-q_{k}^{2}} \\
& =-(-1)^{m} \delta_{m m^{\prime}},
\end{aligned}
$$

where $H_{m}($.$) is a Hermite polynomial of order n$ [57]. This result coincides with the result obtained before for the operator (12). Notice also that $\hat{\mathcal{S}}_{x}$ and $\hat{\mathcal{S}}_{y}$ can be 

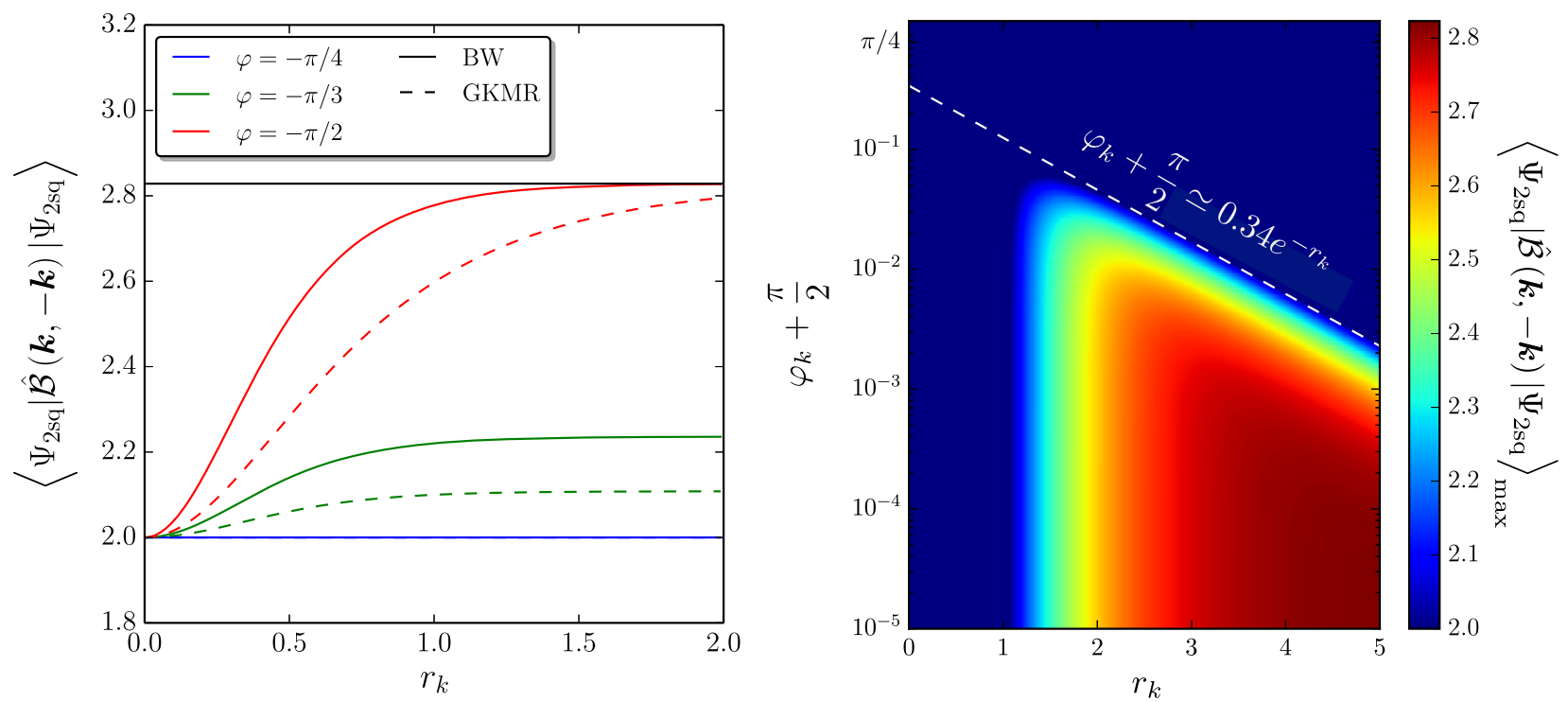

FIG. 2. Left panel: Mean value $\left\langle\Psi_{2 \mathrm{sq}}|\hat{\mathcal{B}}(\boldsymbol{k},-\boldsymbol{k})| \Psi_{2 \mathrm{sq}}\right\rangle$ of the Bell operators for the BW (solid lines, see Sec. III B) and GKMR (dashed lines, see Sec. III C) pseudospin operators, when the system is placed in the two-mode squeezed state (5), as a function of the squeezing parameter $r_{k}$, for different values of the squeezing angle $\varphi_{k}$. The blue line corresponds to $\varphi_{k}=-\pi / 4$, the green lines to $\varphi_{k}=-\pi / 3$ and the red lines to $\varphi=-\pi / 2$. One can check in Eqs. (18) and (26) that $\varphi_{k}=-\pi / 4$ (blue line) indeed leads to a constant $\left\langle\Psi_{2 \mathrm{sq}}|\hat{\mathcal{B}}(\boldsymbol{k},-\boldsymbol{k})| \Psi_{2 \mathrm{sq}}\right\rangle=2$. The horizontal black line represents the Cirel'son bound [54]. Right panel: Maximum Bell operator expectation value $\left\langle\Psi_{2 \mathrm{sq}}|\hat{\mathcal{B}}(\boldsymbol{k},-\boldsymbol{k})| \Psi_{2 \mathrm{sq}}\right\rangle$ for the Larsson pseudospin operators, see Sec. III D, where extremization has been performed over $\ell$, as a function of the squeezing parameters $r_{k}$ and $\varphi_{k}$. The dashed white line stands for $\varphi_{k}+\pi / 2=0.34 e^{-r_{k}}$ which delimits the Bell inequality violation domain in the large-squeezing limit. This figure is adapted from Ref. [32].

written as $\hat{\mathcal{S}}_{x}=\int_{0}^{\infty} \mathrm{d} q_{\boldsymbol{k}}\left(\left|q_{\boldsymbol{k}}\right\rangle\left\langle q_{\boldsymbol{k}}|-|-q_{\boldsymbol{k}}\right\rangle\left\langle-q_{k}\right|\right)$ and $\hat{\mathcal{S}}_{y}=-\int_{0}^{\infty} \mathrm{d} q_{k}\left(\left|q_{k}\right\rangle\left\langle-q_{k}|-|-q_{k}\right\rangle\left\langle q_{k}\right|\right)$.

Then, one can proceed exactly as before, namely introduce a Bell operator and perform the orientational optimization. One then obtains Eq. (18), with $\left\langle\Psi_{2 \mathrm{sq}}\left|\hat{\mathcal{S}}_{z}(\boldsymbol{k}) \otimes \hat{\mathcal{S}}_{z}(-\boldsymbol{k})\right| \Psi_{2 \mathrm{sq}}\right\rangle=1$ and the calculation of Appendix, see Eq. (A13), shows that

$$
\begin{aligned}
& \left\langle\Psi_{2, \mathrm{sq}}\left|\hat{\mathcal{S}}_{x}(\boldsymbol{k}) \hat{\mathcal{S}}_{x}(-\boldsymbol{k})\right| \Psi_{2, \mathrm{sq}}\right\rangle \\
& \quad=\frac{2}{\pi} \times \arctan \left[\frac{2 \tanh \left(r_{k}\right) \cos \left(2 \varphi_{k}\right)}{\sqrt{\tanh ^{4}\left(r_{k}\right)-2 \tanh ^{2}\left(r_{k}\right) \cos \left(4 \varphi_{k}\right)+1}}\right] .
\end{aligned}
$$

This gives the exact dependence of this violation with the squeezing parameters. In the case where $\varphi_{k} \neq 0$, this expression is new. The mean value of the Bell operator as a function of the squeezing parameter, for different values of the squeezing angle, is represented in the left panel of Fig. 2. The violation is slightly less strong than with the BW pseudo-spin operators introduced in Sec. III B but clearly, we have designed another possible situation that leads to a violation of the Bell inequality. On super-Hubble scales in particular, since $r_{k} \rightarrow \infty$ and $\varphi_{k} \rightarrow-\pi / 2$, one has $\left\langle\Psi_{2 \mathrm{sq}}\left|\hat{\mathcal{B}}_{\mathrm{GKMR}}(\boldsymbol{k},-\boldsymbol{k})\right| \Psi_{2 \mathrm{sq}}\right\rangle \rightarrow 2 \sqrt{2}$ and the Cirel'son bound is again saturated.

\section{Larsson spin operators}

So far, we have introduced two different sets of pseudospin operators, and a third one can be defined in the following way $[32,58]$. The idea is to divide the real axis in an infinite number of cells $[n \ell,(n+1) \ell]$ of length $\ell$, where $n$ is an integer number running from $-\infty$ to $+\infty$. The "coarse-grained" parameter $\ell$ is chosen by the observer. Then one introduces the following operator

$$
\hat{S}_{z}(\ell)=\sum_{n=-\infty}^{\infty}(-1)^{n} \int_{n \ell}^{(n+1) \ell} \mathrm{d} q_{k}\left|q_{k}\right\rangle\left\langle q_{k}\right| .
$$

This defines a spin variable because the eigenvalues of this operator are \pm 1 .

The other pseudospin components can be defined by means of the following operators

$$
\begin{aligned}
& \hat{S}_{x}(\ell)=\hat{S}_{+}(\ell)+\hat{S}_{-}(\ell), \\
& \hat{S}_{y}(\ell)=-i\left[\hat{S}_{+}(\ell)-\hat{S}_{-}(\ell)\right],
\end{aligned}
$$

with

$$
\hat{S}_{+}(\ell)=\sum_{n=-\infty}^{\infty} \int_{2 n \ell}^{(2 n+1) \ell} \mathrm{d} q_{k}\left|q_{k}\right\rangle\left\langle q_{k}+\ell\right|
$$

and $\hat{S}_{-}(\ell)=\hat{S}_{+}^{\dagger}(\ell)$. It can be easily shown $[32,58]$ that $\hat{S}_{x}$, $\hat{S}_{y}$ and $\hat{S}_{z}$ satisfy all the properties of a spin operators 
system. It is then sufficient to proceed as before, namely to define a pseudo Bell operator using the spin operators that we have just introduced, perform the orientational optimization and, then, study its mean value in a two-mode squeezed state. In the case of the operators (27), (28), and (29), there does not exist a simple, explicit, analytical expression for the spin correlators, similar to Eqs. (19) and (26). The calculation has therefore to be done numerically. It was carried out in Ref. [32] and, in that paper, it was shown that the Bell inequality is indeed violated in that case. In the right panel of Fig. 2, we have reproduced from Ref. [32] a map of the maximal Bell operator expectation value (where the extremization is performed over $\ell$ ) as a function of the squeezing parameters. We thus have a third example of a design that violates the Bell inequality.

Contrary to the two previous examples however, in the super-Hubble limit, the Cirel'son bound is not necessarily saturated, and the exact value of the Bell operator expectation value depends on the details of the inflationary dynamics. In the large-squeezing limit indeed, in Ref. [32], it was shown that all pseudo-spin correlation functions depend only on the combination (see footnote 1) $\left(\varphi_{k}+\pi / 2\right) e^{r_{k}}$, and Bell inequality violation is obtained when $\left(\varphi_{k}+\pi / 2\right) e^{r_{k}}<0.34$ (see the right panel of Fig. 2 and/or Fig. 16 of Ref. [32]). In the super-Hubble limit, $\varphi_{k}+\pi / 2 \rightarrow 0$ but $e^{r_{k}} \rightarrow \infty$ so a more detailed analysis is required. At leading order in the slow-roll approximation, the Mukhanov-Sasaki variable takes the form $v_{k}(\eta)=$ $\sqrt{\pi / k} / 2 \sqrt{-k \eta} e^{-i \pi(\nu+1 / 2) / 2} H_{\nu}^{(2)}(-k \eta)$ where we recall that $\eta$ is the conformal time, $H_{\nu}^{(2)}$ is the Hankel function of the second kind, and $\nu$ is a constant that can be related to the spectral index $n_{\mathrm{S}}$ of the curvature perturbations power spectrum according to $\nu=3 / 2+\left(n_{\mathrm{S}}-1\right) / 2$, where this expression is valid again at leading order in slow roll. The relationship between the squeezing parameters and the Mukhanov-Sasaki variable can be obtained by combining Eqs. (12), (16), (24) and (25) of Ref. [29] and one obtains

$$
\begin{aligned}
\cosh ^{2} r_{k} & =\frac{1}{8 k v_{\boldsymbol{k}} v_{\boldsymbol{k}}^{*}}\left[\left(v_{\boldsymbol{k}}^{*} v_{\boldsymbol{k}}^{\prime}+v_{\boldsymbol{k}} v_{\boldsymbol{k}}^{*}-2 \frac{z^{\prime}}{z} v_{\boldsymbol{k}} v_{\boldsymbol{k}}^{*}\right)^{2}+\left(1+2 k v_{\boldsymbol{k}} v_{\boldsymbol{k}}^{*}\right)^{2}\right], \\
\tan \left(2 \varphi_{k}\right) & =4 k v_{\boldsymbol{k}} v_{\boldsymbol{k}}^{*} \frac{v_{\boldsymbol{k}}^{*} v_{\boldsymbol{k}}^{\prime}+v_{\boldsymbol{k}} v_{\boldsymbol{k}}^{\prime *}-2 \frac{z^{\prime}}{z} v_{\boldsymbol{k}} v_{\boldsymbol{k}}^{*}}{\left(2 k v_{\boldsymbol{k}} v_{\boldsymbol{k}}^{*}\right)^{2}-1-\left(v_{\boldsymbol{k}}^{*} v_{\boldsymbol{k}}^{\prime}+v_{\boldsymbol{k}} v_{\boldsymbol{k}}^{*}-2 \frac{z^{\prime}}{z} v_{\boldsymbol{k}} v_{\boldsymbol{k}}^{*}\right)^{2}} .
\end{aligned}
$$

Using the fact that, in slow-roll inflation, $z^{\prime} / z \simeq(1 / 2-\nu) / \eta$, in terms of the number of $e$-folds $N-N_{*}(k)$ spent by the mode $k$ outside the Hubble radius, one obtains, in the super-Hubble limit and at leading order in slow roll,

$$
\left(\varphi_{k}+\frac{\pi}{2}\right) e^{r_{k}} \simeq \exp \left\{\frac{1-n_{\mathrm{S}}}{2}\left[N-N_{*}(k)\right]\right\}
$$

In the case of de Sitter $\left(n_{\mathrm{S}}=1\right)$, this combination turns out to be time independent and equal to one, meaning no Bell inequality violation. In the more general case, however, this is clearly a time-dependent quantity. For $\left(\varphi_{k}+\pi / 2\right) e^{r_{k}}$ to be smaller than 0.34 , one can see that the power spectrum has to be blue, $n_{\mathrm{S}}>1$, which is excluded by the data [10]. Therefore, unless slow roll is violated at some point during inflation, the Larsson pseudospin operators are not the best candidates to yield a Bell inequalities violation.

To our knowledge, no other pseudospin operators for continuous variables have been proposed and, therefore, we have now covered all the cases. In the next section, we discuss these results and consider the question of whether these spin operators can really be measured on the sky.

\section{DISCUSSION}

\section{A. Bell inequality violation and the positivity of the Wigner function}

Let us start by a remark about the consistency of the previous results where Bell inequality violations are obtained with a non-negative Wigner function. We notice that some of the pseudospin operators we have introduced are improper dynamical variables, a notion that we now explain with an example (a more complete discussion can be found in Ref. [53]). Let $\hat{A}=\mathcal{A}\left(\hat{q}_{k}, \hat{p}_{k}\right)$ be a general operator (we restrict ourselves to a one-dimensional phase space but the generalization to higher dimensional phase spaces is trivial), where we use the position and momentum introduced before. Its Wigner-Weyl representation is defined by

$W_{\hat{A}}\left(q_{k}, \pi_{k}\right) \equiv \int_{\mathbb{R}} \mathrm{d} x e^{-i \pi_{k} x}\left\langle q+\frac{x}{2}\left|\mathcal{A}\left(\hat{q}_{k}, \hat{p}_{k}\right)\right| q_{k}-\frac{x}{2}\right\rangle$.

This is clearly a function (i.e. not an operator) on phase space. Let us now consider a (bounded) function $\mathcal{F}($.) defined on the real axis $\mathbb{R}$. We can then introduce the following operator 


$$
\hat{F} \equiv \int_{-\infty}^{+\infty} \mathrm{d} q_{k}^{\prime}\left|q_{k}^{\prime}\right\rangle \mathcal{F}\left(q_{k}^{\prime}\right)\left\langle q_{k}^{\prime}\right| .
$$

The eigenvalues of the operator $\hat{F}$ are precisely $\mathcal{F}(\mathbb{R})$ (this is a way to define an operator from its spectrum). Then, it is a trivial calculation to show that

$$
W_{\hat{F}}\left(q_{k}, \pi_{k}\right)=\mathcal{F}\left(q_{k}\right) .
$$

So the function $W_{\hat{F}}(q, \pi)$ takes all and only the eigenvalues of the operator $\hat{F}$. We then say that $\hat{F}$ is a proper dynamical variable. Of course, the previous calculation is only an illustrative example and can easily be generalized, for instance to functions of $\pi_{k}$, see Ref. [53].

Now, it is easy to show that the pseudospin operators that we have introduced above are not proper dynamical variables. For instance, the Wigner-Weyl representative of the BW operator $\hat{s}_{z}$ given by Eq. (12) can be expressed as

$$
\begin{aligned}
W_{\hat{s}_{z}}\left(q_{\boldsymbol{k}}, \pi_{\boldsymbol{k}}\right) & \equiv \int_{-\infty}^{+\infty} e^{-i \pi_{k} x} \mathrm{~d} x\left\langle q_{\boldsymbol{k}}+\frac{x}{2}\left|\hat{s}_{z}\right| q_{\boldsymbol{k}}-\frac{x}{2}\right\rangle \\
& =-\pi \delta\left(q_{\boldsymbol{k}}\right) \delta\left(\pi_{\boldsymbol{k}}\right) .
\end{aligned}
$$

Clearly, the function $W_{\hat{s}_{z}}\left(q_{k}, \pi_{k}\right)$ does not consist of only two values \pm 1 .

For the GKMR spin operators defined by Eqs. (22), (23), and (24), we reach the same conclusions, since we have shown that the GKMR operator (24) is in fact the same as the BW operator (12). The Wigner-Weyl representation of $\hat{\mathcal{S}}_{x}$ given by Eq. (22) can be written as

$$
W_{\hat{\mathcal{S}}_{x}}\left(q_{k}, \pi_{k}\right)=\operatorname{sign}\left(q_{k}\right),
$$

i.e. it is a proper dynamical variable according to the previous definition. Finally, the Wigner-Weyl representation of the GKMR $y$-component $(23)$ is $W_{\hat{\mathcal{S}}_{y}}\left(q_{k}, \pi_{k}\right)=$ $-\delta\left(q_{k}\right) \mathcal{P}\left(1 / \pi_{k}\right)$, where $\mathcal{P}$ denotes the principal value and therefore the operator (23) is not proper.

It is also interesting to calculate the Wigner-Weyl transform of the Larsson pseudo-spin operators (28), (29), and (27). Straightforward manipulations lead to

$$
\begin{aligned}
& W_{\hat{S}_{x}}\left(q_{k}, \pi_{k}\right)=2 \sum_{n=-\infty}^{+\infty} \cos \left(\pi_{k} \ell\right)\left[\Theta\left(q_{k}-n \ell-\frac{\ell}{2}\right)-\Theta\left(q_{k}-n \ell-\frac{3}{2} \ell\right)\right], \\
& W_{\hat{S}_{y}}\left(q_{k}, \pi_{k}\right)=2 \sum_{n=-\infty}^{+\infty} \sin \left(\pi_{k} \ell\right)\left[\Theta\left(q_{k}-n \ell-\frac{\ell}{2}\right)-\Theta\left(q_{k}-n \ell-\frac{3}{2} \ell\right)\right], \\
& W_{\hat{S}_{z}}\left(q_{k}, \pi_{k}\right)=\sum_{n=-\infty}^{+\infty}(-1)^{n}\left[\Theta\left(q_{k}-n \ell\right)-\Theta\left(q_{k}-n \ell-\ell\right)\right],
\end{aligned}
$$

where $\Theta($.$) is the Heaviside function. The above equations$ show that $\hat{S}_{z}$ is a proper variable but $\hat{S}_{x}$ and $\hat{S}_{y}$ are not.

We conclude that each of the three sets of pseudospin operators introduced before contains, at least, one improper variable. And this makes perfect sense since there is a theorem stating that, if the Wigner function is positive definite, a violation of the Bell inequality can only occur for improper dynamical variables, see Ref. [53] for a more accurate discussion of this point. This is clearly relevant for the CMB case since, as already mentioned, a two-mode squeezed state has a positive definite Wigner function. In fact, the CMB is a prototypical situation where this theorem is useful.

This however says nothing about the measurability of the effect, which we now discuss.

\section{B. Are the pseudospin operators measurable?}

Answering the question asked in the title of this subsection is not an easy task since it involves the measurement problem of quantum mechanics in the context of cosmology. It seems however reasonable to assume that the temperature anisotropy operator,

$$
\frac{\widehat{\delta T}}{T}(\theta, \phi)=\sum_{\ell=2}^{+\infty} \sum_{m=-\ell}^{m=\ell} \hat{a}_{\ell m} Y_{\ell m}(\theta, \phi),
$$

is an observable, since it is a real quantity. More precisely, it is a family of operators parametrized by the continuous labels $\theta$ and $\phi$. In the above expression, the coefficients $\hat{a}_{\ell m}$ are a collection of, non-Hermitian, operators, as needed for the consistency of this equation. Let us now try to relate the temperature fluctuation operator to the curvature operator $\hat{\zeta}_{k}$ introduced before. The Sachs-Wolfe effect implies that

$$
\begin{aligned}
\frac{\delta T}{T}(\boldsymbol{e})= & \int \frac{\mathrm{d} \boldsymbol{k}}{(2 \pi)^{3 / 2}}[F(\boldsymbol{k})+i \boldsymbol{k} \cdot \boldsymbol{e} G(\boldsymbol{k})] \\
& \times e^{-i \boldsymbol{k} \cdot \boldsymbol{e}\left(\eta_{\mathrm{lss}}-\eta_{0}\right)+i \boldsymbol{k} \cdot x_{0}}
\end{aligned}
$$


where $\boldsymbol{e}$ is a unit vector in the direction labeled by the angles $\theta$ and $\phi$. The quantities $\eta_{\mathrm{lss}}$ and $\eta_{0}$ are the last scattering surface (lss) and present day (0) conformal times while $\boldsymbol{x}_{0}$ represents Earth's location. The functions $F(\boldsymbol{k})$ and $G(\boldsymbol{k})$ are the form factors and describe the evolution of the perturbation in the post-inflationary universe. The important property is that these form factors are proportional to $\zeta_{k}\left(\eta_{\text {end }}\right)$ evaluated at the end of inflation. Strictly speaking, they also depend on the derivative of $\zeta_{k}$ but the point is that this dependence is completely negligible since it is related to the presence of a decaying mode. However, in principle, $\zeta_{k}^{\prime}$ is present. This means that, at the operator level, one can write

$$
\begin{aligned}
\frac{\widehat{\delta T}}{T}(\boldsymbol{e})= & \int \frac{\mathrm{d} \boldsymbol{k}}{(2 \pi)^{3 / 2}}[F(\boldsymbol{k})+i \boldsymbol{k} \cdot \boldsymbol{e} G(\boldsymbol{k})] \hat{\zeta}_{\boldsymbol{k}}\left(\eta_{\mathrm{end}}\right) \\
& \times e^{-i \boldsymbol{k} \cdot \boldsymbol{e}\left(\eta_{\mathrm{lss}}-\eta_{0}\right)+i \boldsymbol{k} \cdot \boldsymbol{x}_{0}},
\end{aligned}
$$

where, compared to Eq. (41), we have slightly redefined the form factors. In particular, the above expression implies that $\widehat{\delta T / T}$ for two different directions $\boldsymbol{e}$ and $\boldsymbol{e}^{\prime}$ are commuting operators since $\left[\hat{\zeta}_{k}, \hat{\zeta}_{p}\right]=0$. Notice that this result crucially rests on the fact that we have neglected the decaying mode. If not, the temperature anisotropy would depend on $\zeta_{k}^{\prime}$, namely on the momentum and, therefore, it would no longer commute for different directions on the sky. We also conclude that the eigenvectors of $\widehat{\delta T / T}(\theta, \phi)$, that we denote $|\varnothing\rangle$ since a measurement of the CMB temperature anisotropies projects the state to one of these eigenvectors, are those of $\hat{\zeta}_{k}$. Then, given the fact that $\hat{\zeta}_{k}=\left(c_{k}+c_{-k}^{\dagger}\right) / \sqrt{2 k}$, one can write the state $|\odot\rangle$ in terms of the eigenvectors of the creation and annihilation operators, namely the coherent states. This leads to

$$
|\varnothing\rangle=e^{-\frac{1}{2}\left(\left|\alpha_{\boldsymbol{k}}\right|^{2}+\left|\alpha_{-\boldsymbol{k}}\right|^{2}\right)} \sum_{n, m} \frac{\alpha_{\boldsymbol{k}}^{n}\left(\alpha_{-\boldsymbol{k}}^{*}\right)^{m}}{\sqrt{n ! m !}}\left|n_{\boldsymbol{k}}, m_{-\boldsymbol{k}}\right\rangle .
$$

This also implies that the two-mode squeezed state introduced before is not an eigenstate of the temperature anisotropy operator, which means that

$$
\left|\Psi_{2 \mathrm{sq}}\right\rangle=\sum c(\ominus)|\odot\rangle \text {. }
$$

Since we observe a specific map, one has then to assume that, after our "observation" of the sky, the system is placed in the "eigenstate" $|\varnothing\rangle$ corresponding to the "eigenvalue" $\delta T /\left.T(\theta, \phi)\right|_{\text {Planck }}$, namely the Planck map. How the process

$$
\left|\Psi_{2 \mathrm{sq}}\right\rangle=\sum c(\odot)|\odot\rangle \rightarrow|\odot\rangle_{\text {Planck }}
$$

occurred is of course the quantum measurement problem which is usually "solved" by the collapse postulate. However, the status of this postulate is, to say the least, unclear in cosmology and alternatives to the standard Copenhagen interpretation have been proposed such as, continuous spontaneous localization (CSL) models [59-66], the manyworlds $[67,68]$ or the Bohm-de Broglie interpretations [69-72]. Here, we will not attempt to discuss these issues and will just assume that the system is placed in a specific eigenvector corresponding to a specific eigenvalue, namely the Planck map $|\odot\rangle$.

Let us now see what it means to "measure" the spin operators. According to the previous discussion, it seems reasonable to assume that we have measured $\hat{\zeta}_{k}$, i.e. we have a collection of numbers $\zeta_{k}$. Of course, one can only measure real quantities and, therefore, one should rather say that we have measured the real and imaginary parts of the operators $\hat{\zeta}_{k}$. But this is equivalent since all these quantities commute and, therefore, a measurement of the real and imaginary parts of the curvature perturbations is also a measurement of $\hat{\zeta}_{k}$ and its Hermitian conjugate, exactly as a measurement of $\hat{\mathcal{O}}$ is also a measurement of any function $f(\hat{\mathcal{O}})$ since $[\hat{\mathcal{O}}, f(\hat{\mathcal{O}})]=0$ [if we have found $\hat{\mathcal{O}}$ to be the number $o$, then, because of their vanishing commutator, we are entitled to say that we have measured $f(\hat{\mathcal{O}})$ to be $f(o)]$. Then, using Eqs. (2) and (3), it is easy to establish that

$$
\hat{q}_{k}=\frac{z}{2}\left(\hat{\zeta}_{k}+\hat{\zeta}_{-k}\right)+\frac{z}{2 k}\left(\zeta_{k}^{\prime}-\zeta_{-k}^{\prime}\right),
$$

and we see that the knowledge of $\hat{\zeta}_{k}$ is not sufficient to infer $\hat{q}_{k}$. However, if the decaying mode is neglected, then a measurement of $\hat{\zeta}_{k}$ is a measurement of the operator $\hat{q}_{k}$. Notice that in this limit, $\hat{q}_{\boldsymbol{k}}=\hat{q}_{-\boldsymbol{k}}$, hence $\hat{\mathcal{S}}_{x}(\boldsymbol{k})=\hat{\mathcal{S}}_{x}(-\boldsymbol{k})$ [since they share the same Wigner-Weyl representation according to Eq. (38)], thus $\hat{\mathcal{S}}_{x}(\boldsymbol{k}) \otimes \hat{\mathcal{S}}_{x}(-\boldsymbol{k})=\hat{\mathrm{I}}$. As a consequence, $\left\langle\Psi_{2 \text { sq }}\left|\hat{\mathcal{S}}_{x}(\boldsymbol{k}) \otimes \hat{\mathcal{S}}_{x}(-\boldsymbol{k})\right| \Psi_{2 \mathrm{sq}}\right\rangle=1$, which is consistent with the large-squeezing limit of Eq. (26). Moreover, since $\left\langle\Psi_{2 \text { sq }}\left|\hat{\mathcal{S}}_{z}(\boldsymbol{k}) \otimes \hat{\mathcal{S}}_{z}(-\boldsymbol{k})\right| \Psi_{2 \mathrm{sq}}\right\rangle$ is always 1 , this also shows, as announced in the introduction, that a Bell inequality violation (furthermore a maximal one) can be obtained even when the decaying mode is neglected.

In the following, we assume that the decaying mode can be ignored and study the consequences. The question is now, given the knowledge of the numbers $q_{k}$, can we infer the values of the pseudospin operators? Let us first discuss the BW spin operators defined by Eqs. (10), (11), and (12). One can show that

$$
\hat{s}_{z}(\boldsymbol{k})=-(-1)^{\hat{N}_{k}}=-\int_{-\infty}^{+\infty} \mathrm{d} q_{\boldsymbol{k}}\left|q_{\boldsymbol{k}}\right\rangle\left\langle-q_{\boldsymbol{k}}\right|,
$$

where we recall that $\hat{N}_{\boldsymbol{k}}=\hat{c}_{\boldsymbol{k}}^{\dagger} \hat{c}_{\boldsymbol{k}}$ is the particle number operator. This operator does not commute with $\hat{q}_{k}$ since one has 


$$
\begin{aligned}
\left\langle q_{k}\left|\left[\hat{s}_{z}(\boldsymbol{k}), \hat{q}_{k}\right]\right| q_{k}^{\prime}\right\rangle & =\left(q_{k}-q_{k}^{\prime}\right)\left\langle-q_{k} \mid q_{k}^{\prime}\right\rangle \\
& =2 q_{k} \delta\left(q_{k}+q_{k}^{\prime}\right) \neq 0 .
\end{aligned}
$$

Since, in cosmology, we are given a measurement and, contrary to what happens in the laboratory, we cannot perform a new measurement, this means that one simply cannot measure $\hat{s}_{z}(\boldsymbol{k})$ if $\hat{q}_{\boldsymbol{k}}$ has been measured. On the other hand, the operators $\hat{s}_{x}$ and $\hat{s}_{y}$ are related to the parity flip operators $\hat{s}_{ \pm}$through $\hat{s}_{ \pm} \equiv\left(\hat{s}_{x} \pm i \hat{s}_{y}\right) / 2$, where

$$
\hat{s}_{-}(\boldsymbol{k})=\left[\hat{\mathrm{I}}+(-1)^{\hat{N}_{k}}\right] \frac{1}{2 \sqrt{\hat{N}_{k}+1}} \hat{c}_{k},
$$

and $\hat{s}_{+}=\hat{s}_{-}^{\dagger}$. It is not obvious to design an experimental protocol in order to measure these operators. It is not even clear whether this is, in principle, feasible. In any case, since the operator $\hat{c}_{k}$ depends on $\hat{q}_{k}$ and $\hat{\pi}_{k}$, it is hard to see how $\hat{s}_{ \pm}$could commute with $\hat{q}_{k}$. So, as far as the BM spins defined by Eqs. (10), (11), and (12) are concerned, we are in a situation where it is probably impossible to infer their values from the data.

The same conclusion is also valid for the GKMR operators defined in Eqs. (22), (23), and (24) since, for instance, they share the same $z$-component spin operator as the one of the BW proposal, namely $\hat{s}_{z}=\hat{\mathcal{S}}_{z}$. However, it is interesting to notice that

$$
\left\langle q_{k}\left|\left[\hat{\mathcal{S}}_{x}(\boldsymbol{k}), \hat{q}_{k}\right]\right| q_{\boldsymbol{k}}^{\prime}\right\rangle=0,
$$

which means that $\hat{\mathcal{S}}_{x}(\boldsymbol{k})$ can be measured. In fact, it is also easy to show that

$$
\hat{\mathcal{S}}_{x}\left|q_{k}\right\rangle=\operatorname{sign}\left(q_{k}\right)\left|q_{k}\right\rangle,
$$

which means that, once we are given the number $q_{k}$, the value of $\hat{\mathcal{S}}_{x}$ is just the sign of $q_{\boldsymbol{k}}$. Of course, this is compatible with its Weyl-Wigner representation, see Eq. (38). The fact that $\hat{\mathcal{S}}_{x}(\boldsymbol{k})$ can be measured does not "save" the GKMR operators since we would need another measurable operator, which is not the case for the only one left, namely $\hat{\mathcal{S}}_{y}$, since

$$
\begin{aligned}
\left\langle q_{\boldsymbol{k}}\left|\left[\hat{\mathcal{S}}_{y}(\boldsymbol{k}), \hat{q}_{\boldsymbol{k}}\right]\right| q_{\boldsymbol{k}}^{\prime}\right\rangle= & \left(q_{\boldsymbol{k}}-q_{\boldsymbol{k}}^{\prime}\right)\left\langle-q_{\boldsymbol{k}} \mid q_{\boldsymbol{k}}^{\prime}\right\rangle+q_{\boldsymbol{k}}^{\prime}\left\langle q_{\boldsymbol{k}} \mid-q_{\boldsymbol{k}}^{\prime}\right\rangle \\
& +q_{\boldsymbol{k}}\left\langle q_{\boldsymbol{k}} \mid q_{\boldsymbol{k}}^{\prime}\right\rangle \\
= & q_{\boldsymbol{k}}\left[\delta\left(q_{\boldsymbol{k}}+q_{\boldsymbol{k}}^{\prime}\right)+\delta\left(q_{\boldsymbol{k}}-q_{\boldsymbol{k}}^{\prime}\right)\right] \neq 0 .
\end{aligned}
$$

Finally remains the Larsson operators (28), (29), and (27). In particular, the $\hat{S}_{z}$ operator is measurable since one has

$$
\left\langle q_{k}\left|\left[\hat{S}_{z}(\boldsymbol{k}), \hat{q}_{k}\right]\right| q_{k}^{\prime}\right\rangle=0
$$

Indeed, it is easy to show that

$\hat{S}_{z}\left|q_{k}\right\rangle=\sum_{n=-\infty}^{n=\infty}(-1)^{n}\left[\Theta\left(q_{k}-n \ell\right)-\Theta\left(q_{k}-n \ell-\ell\right)\right]\left|q_{k}\right\rangle$.

This formula tells us that, in practice, the observer chooses a value of $\ell$ and, given a measurement of $\hat{q}_{k}$, identifies the value of $n$ such that $q_{k} \in[n \ell,(n+1) \ell]$. The measurement of $\hat{S}_{z}(\ell)$ is then $(-1)^{n}$. However, neither $\hat{S}_{x}(\ell)$ nor $\hat{S}_{y}(\ell)$ can be inferred from the knowledge of $q_{k}$ since

$$
\left\langle q_{k}\left|\left[\hat{S}_{x}(\boldsymbol{k}), \hat{q}_{k}\right]\right| q_{\boldsymbol{k}}^{\prime}\right\rangle \neq 0,\left\langle q_{\boldsymbol{k}}\left|\left[\hat{S}_{y}(\boldsymbol{k}), \hat{q}_{\boldsymbol{k}}\right]\right| q_{\boldsymbol{k}}^{\prime}\right\rangle \neq 0,
$$

as can be established from the result

$$
\begin{aligned}
\left\langle q_{k}\left|\left[\hat{S}_{+}(\boldsymbol{k}), \hat{q}_{k}\right]\right| q_{k}^{\prime}\right\rangle= & \ell \sum_{n=-\infty}^{n=+\infty}\left[\Theta\left(q_{k}-2 n \ell\right)\right. \\
& \left.-\Theta\left(q_{k}-2 n \ell-\ell\right)\right] \delta\left(q_{k}^{\prime}-q_{k}-\ell\right),
\end{aligned}
$$

$$
\begin{aligned}
\left\langle q_{k}\left|\left[\hat{S}_{-}(\boldsymbol{k}), \hat{q}_{k}\right]\right| q_{\boldsymbol{k}}^{\prime}\right\rangle= & -\ell \sum_{n=-\infty}^{n=+\infty}\left[\Theta\left(q_{k}-2 n \ell-\ell\right)\right. \\
& \left.-\Theta\left(q_{k}-2 n \ell-2 \ell\right)\right] \delta\left(q_{\boldsymbol{k}}^{\prime}-q_{k}+\ell\right) .
\end{aligned}
$$

We conclude this section by stressing out that the knowledge of the numbers $q_{k}$ is not sufficient to determine two spin operators, which is necessary to observe a Bell inequality violation in the data. This limitation seems to be deeply rooted in the fact that we work in a cosmological context. Indeed, in a conventional situation, one would first measure, say, the $z$-component of the spin and, then, in a second time, one would repeat the experiment and measure, say, the $x$-component. In cosmology, one cannot repeat the experiment and, in some sense, we are given the measurement. This means that we are unable to determine the value of two noncommuting observables from the data. In the laboratory, this would be like being given a measure of the $z$-component only and trying to infer a Bell inequality violation from this single measurement. A possible way out would be to use a kind of ergodic theorem. Indeed, as we have already seen, $\widehat{\delta T / T}(\theta, \phi)$ is in fact a collection of operators, one for each direction in the sky. One could then imagine to perform a measurement of the $x$-component in one direction and of the $y$-component in another direction. However, at least if the Copenhagen interpretation is taken to its logical extreme, this is already "too late", since we have already measured the operator $\hat{q}_{k}$ (or $\hat{\zeta}_{k}$ ) over the entire celestial sphere. Therefore, at this point, it seems that there is no hope to observe a Bell inequality violation in the 


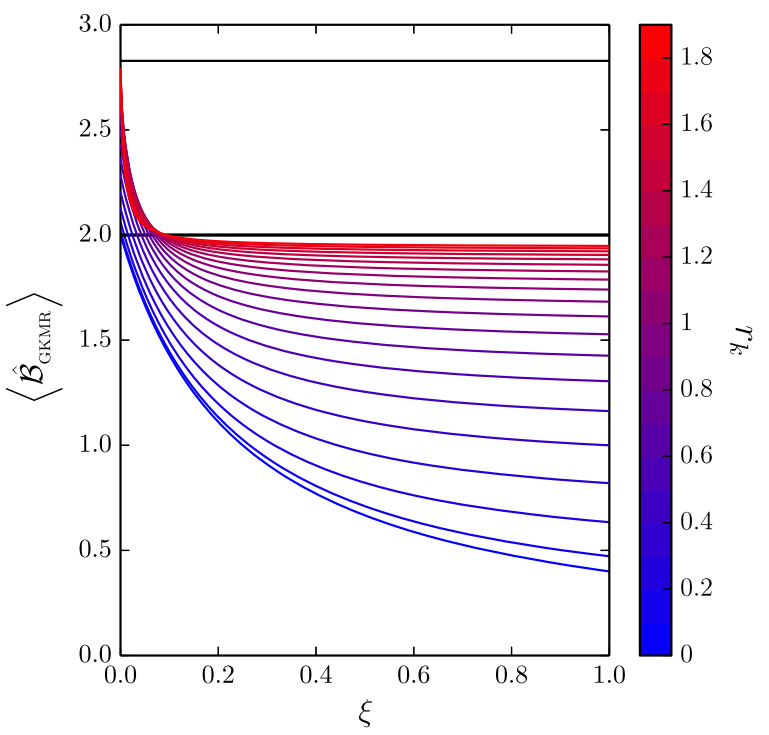

FIG. 3. Mean value of the Bell operator for the GKMR pseudospin operators in presence of decoherence parametrized by the quantity $\xi$. Different values of the squeezing parameter $r_{k}$ are displayed with different colors, and the squeezing angle is chosen to be $\varphi_{k}=-\pi / 2$.

CMB data unless we find a way to go beyond these fundamental limitations.

\section{Decoherence}

It is also interesting to study how robust our results are against quantum decoherence [73-78]. For this purpose, we model decoherence through the generic Gaussian channel which consists in replacing the density matrix $\rho$ according to [75] $\rho\left(q_{k}, q_{-k}, \tilde{q}_{k}, \tilde{q}_{-k}\right) \rightarrow$ $\rho\left(q_{k}, q_{-k}, \tilde{q}_{k}, \tilde{q}_{-k}\right) \exp \left[-\xi\left(q_{k}-\tilde{q}_{k}\right)^{2}-\xi\left(q_{-k}-\tilde{q}_{-k}\right)^{2}\right]$, where the phenomenological parameter $\xi$ encodes the interaction strength with the environment. Given a concrete model for the environement, in principle, one could determine $\xi$ explicitly. We illustrate the effect of decoherence with the GKMR pseudospin operators (22)-(24). As already mentioned, the mean value of the Bell operator is given by an equation similar to Eq. (18), where $\left\langle\Psi_{2 \text { sq }}\right| \hat{\mathcal{S}}_{x}(\boldsymbol{k}) \otimes$ $\hat{\mathcal{S}}_{x}(-\boldsymbol{k})\left|\Psi_{2 \mathrm{sq}}\right\rangle$ is still given by Eq. (26), while, instead of $\left\langle\Psi_{2 \mathrm{sq}}\left|\hat{\mathcal{S}}_{z}(\boldsymbol{k}) \otimes \hat{\mathcal{S}}_{z}(-\boldsymbol{k})\right| \Psi_{2 \mathrm{sq}}\right\rangle=1$, one now has

$$
\begin{aligned}
& \left\langle\Psi_{2 \mathrm{sq}}\left|\hat{\mathcal{S}}_{z}(\boldsymbol{k}) \otimes \hat{\mathcal{S}}_{z}(-\boldsymbol{k})\right| \Psi_{2 \mathrm{sq}}\right\rangle=\left[1+8 \xi \cosh \left(2 r_{k}\right)\right. \\
& \left.\quad+12 \xi^{2}+4 \xi^{2} \cos \left(4 \varphi_{k}\right)+8 \xi^{2} \cosh \left(4 r_{k}\right) \sin ^{2}\left(2 \varphi_{k}\right)\right]^{-1 / 2} .
\end{aligned}
$$

The corresponding Bell operator mean value is shown in Fig. 3. As expected, one can see that as $\xi$ increases, coherence is lost, the mean value of $\hat{B}_{\mathrm{GKMR}}$ becomes smaller than 2 and Bell inequality violation no longer exists. For large values of $r_{k}$, the disappearance of Bell inequality violation occurs rapidly, even though $\left\langle\hat{B}_{\mathrm{GKMR}}\right\rangle$ remains close to (while being less than) the Bell violation threshold 2. This is a consequence of the well-known fragility of highly-squeezed states under environmental influence [79]. This result suggests that decoherence is another challenge for Bell CMB experiments.

\section{CONCLUSIONS}

Inflation implies that the quantum state of CMB anisotropies is a two-mode squeezed state. This state is an entangled state and, as a consequence, it is natural to imagine that a cosmic version of the Bell experiment could reveal quantum correlations in the sky. In this article, we have discussed the problems one faces when one tries to implement this program concretely. We have exhibited different possible setups and identified those which, in principle, could be realized. However, we have also shown that all of them are such that only one component of the pseudospin operators can at most, in practice, be inferred from the data, which is not sufficient to observe a Bell inequality violation. In some sense, we encounter again the well-known problem that the quantum behavior of the perturbations is hidden in the decaying mode, but more crucially, we also face the fact that one cannot perform repeated measurements of the CMB along different spin operators, as is necessary in standard Bell experiments. The approach followed in this article attempts to circumvent these problems from a completely new perspective and, therefore, sheds new light on these questions. In particular, it shows that the only way out is to design a method which makes use of only one pseudospin component, either $\hat{\mathcal{S}}_{x}$ or $\hat{S}_{z}$. This turns out to be possible in the Leggett-Garg inequality $[80,81]$ proposal where the same spin component is measured at different times. Since it was recently shown [82] that the Leggett-Garg inequality is violated if the state of the system is a squeezed state, this opens up another possibility for hunting down the quantum origin of the cosmological structures about which we plan to report soon.

\section{ACKNOWLEDGMENTS}

V. V. acknowledges funding from the European Union's Horizon 2020 research and innovation programme under the Marie Skłodowska-Curie Grant Agreement No. 750491 and financial support from Science and Technology Facilities Council (STFC) Grants No. ST/K00090X/1 and No. ST/N000668/1.

\section{APPENDIX: CORRELATION FUNCTION OF $\hat{\mathcal{S}}_{x}$}

In this appendix, we show how to arrive at Eq. (26). The $\hat{\mathcal{S}}_{x}(\boldsymbol{k})$ operator defined in Eq. (22) can also be written as 


$$
\hat{\mathcal{S}}_{x}(\boldsymbol{k})=\int_{0}^{+\infty} \mathrm{d} \tilde{q}_{\boldsymbol{k}}\left(\left|\tilde{q}_{\boldsymbol{k}}\right\rangle\left\langle\tilde{q}_{\boldsymbol{k}}|-|-\tilde{q}_{\boldsymbol{k}}\right\rangle\left\langle-\tilde{q}_{\boldsymbol{k}}\right|\right) .
$$

The action of $\hat{\mathcal{S}}_{x}(-\boldsymbol{k})$ on the two-mode squeezed state (5) can be expressed as

$$
\begin{aligned}
\hat{\mathcal{S}}_{x}(-\boldsymbol{k})\left|\Psi_{2, \mathrm{sq}}\right\rangle= & \int_{0}^{+\infty} \mathrm{d} \tilde{q}_{-\boldsymbol{k}} \sum_{n=0}^{+\infty} \frac{1}{\cosh r_{k}} e^{-2 i n \varphi_{k} \tanh ^{n} r_{k}} \\
& \times\left(\left\langle\tilde{q}_{-\boldsymbol{k}} \mid n_{-\boldsymbol{k}}\right\rangle\left|n_{\boldsymbol{k}}, \tilde{q}_{-\boldsymbol{k}}\right\rangle\right. \\
& \left.-\left\langle-\tilde{q}_{-\boldsymbol{k}} \mid n_{-\boldsymbol{k}}\right\rangle\left|n_{\boldsymbol{k}},-\tilde{q}_{-\boldsymbol{k}}\right\rangle\right) .
\end{aligned}
$$

Then, the next step is to apply the operator $\hat{\mathcal{S}}_{x}(\boldsymbol{k})$ on the previous state. This leads to

$$
\begin{aligned}
\hat{\mathcal{S}}_{x}(\boldsymbol{k}) \hat{\mathcal{S}}_{x}(-\boldsymbol{k})\left|\Psi_{2, \mathrm{sq}}\right\rangle= & \int_{0}^{+\infty} \int_{0}^{+\infty} \mathrm{d} \tilde{q}_{-\boldsymbol{k}} \mathrm{d} q_{\boldsymbol{k}} \sum_{n=0}^{+\infty} \frac{1}{\cosh r_{k}} e^{-2 i n \varphi_{k} \tanh ^{n} r_{k}\left(\left\langle\tilde{q}_{-\boldsymbol{k}} \mid n_{-\boldsymbol{k}}\right\rangle\left\langle q_{\boldsymbol{k}} \mid n_{\boldsymbol{k}}\right\rangle\left|q_{\boldsymbol{k}}, \tilde{q}_{-\boldsymbol{k}}\right\rangle\right.} \\
& -\left\langle-\tilde{q}_{-\boldsymbol{k}} \mid n_{-\boldsymbol{k}}\right\rangle\left\langle q_{\boldsymbol{k}} \mid n_{\boldsymbol{k}}\right\rangle\left|q_{\boldsymbol{k}},-\tilde{q}_{-\boldsymbol{k}}\right\rangle-\left\langle\tilde{q}_{-\boldsymbol{k}} \mid n_{-\boldsymbol{k}}\right\rangle\left\langle-q_{\boldsymbol{k}} \mid n_{\boldsymbol{k}}\right\rangle\left|-q_{\boldsymbol{k}}, \tilde{q}_{-\boldsymbol{k}}\right\rangle \\
& \left.+\left\langle-\tilde{q}_{-\boldsymbol{k}} \mid n_{-\boldsymbol{k}}\right\rangle\left\langle-q_{\boldsymbol{k}} \mid n_{\boldsymbol{k}}\right\rangle\left|-q_{\boldsymbol{k}},-\tilde{q}_{-\boldsymbol{k}}\right\rangle\right),
\end{aligned}
$$

and, therefore, using again the expression of the two-mode squeezed state, one arrives at

$$
\begin{aligned}
\left\langle\Psi_{2, \mathrm{sq}}\left|\hat{\mathcal{S}}_{x}(\boldsymbol{k}) \hat{\mathcal{S}}_{x}(-\boldsymbol{k})\right| \Psi_{2, \mathrm{sq}}\right\rangle= & \frac{1}{\cosh ^{2} r_{k}} \sum_{n=0}^{+\infty} \sum_{m=0}^{+\infty} e^{-2 i n \varphi_{k}+2 i m \varphi_{k}} \tanh ^{n} r_{k} \tanh ^{m} r_{k} \int_{0}^{+\infty} \int_{0}^{+\infty} \mathrm{d} \tilde{q}_{-\boldsymbol{k}} \mathrm{d} q_{\boldsymbol{k}} \\
& \times\left(\left\langle\tilde{q}_{-\boldsymbol{k}} \mid n_{-\boldsymbol{k}}\right\rangle\left\langle q_{\boldsymbol{k}} \mid n_{\boldsymbol{k}}\right\rangle\left\langle m_{\boldsymbol{k}} \mid q_{\boldsymbol{k}}\right\rangle\left\langle m_{-\boldsymbol{k}} \mid \tilde{q}_{-\boldsymbol{k}}\right\rangle-\left\langle-\tilde{q}_{-\boldsymbol{k}} \mid n_{-\boldsymbol{k}}\right\rangle\left\langle q_{\boldsymbol{k}} \mid n_{\boldsymbol{k}}\right\rangle\left\langle m_{\boldsymbol{k}} \mid q_{\boldsymbol{k}}\right\rangle\left\langle m_{-\boldsymbol{k}} \mid-\tilde{q}_{-\boldsymbol{k}}\right\rangle\right. \\
& \left.-\left\langle\tilde{q}_{-\boldsymbol{k}} \mid n_{-\boldsymbol{k}}\right\rangle\left\langle-q_{\boldsymbol{k}} \mid n_{\boldsymbol{k}}\right\rangle\left\langle m_{\boldsymbol{k}} \mid-q_{\boldsymbol{k}}\right\rangle\left\langle m_{-\boldsymbol{k}} \mid \tilde{q}_{-\boldsymbol{k}}\right\rangle+\left\langle-\tilde{q}_{-\boldsymbol{k}} \mid n_{-\boldsymbol{k}}\right\rangle\left\langle-q_{\boldsymbol{k}} \mid n_{\boldsymbol{k}}\right\rangle\left\langle m_{\boldsymbol{k}} \mid-q_{\boldsymbol{k}}\right\rangle\left\langle m_{-\boldsymbol{k}} \mid-\tilde{q}_{-\boldsymbol{k}}\right\rangle\right) .
\end{aligned}
$$

One can then express explicitly each of the matrix element present in the above equation, obtaining the following expression

$$
\begin{aligned}
& \left\langle\Psi_{2, \mathrm{sq}}\left|\hat{\mathcal{S}}_{x}(\boldsymbol{k}) \hat{\mathcal{S}}_{x}(-\boldsymbol{k})\right| \Psi_{2, \mathrm{sq}}\right\rangle=\frac{1}{\cosh ^{2} r_{k}} \sum_{n=0}^{+\infty} \sum_{m=0}^{+\infty} e^{-2 i n \varphi_{k}+2 i m \varphi_{k}} \tanh ^{n} r_{k} \tanh ^{m} r_{k} \frac{1}{\sqrt{\pi} 2^{n} n !} \frac{1}{\sqrt{\pi} 2^{m} m !} \int_{0}^{+\infty} \int_{0}^{+\infty} \mathrm{d} \tilde{q}_{-\boldsymbol{k}} \mathrm{d} q_{\boldsymbol{k}} \\
& \times\left[H_{n}\left(\tilde{q}_{-k}\right) H_{n}\left(q_{k}\right) H_{m}\left(q_{k}\right) H_{m}\left(\tilde{q}_{-k}\right)-H_{n}\left(-\tilde{q}_{-k}\right) H_{n}\left(q_{k}\right) H_{m}\left(q_{k}\right) H_{m}\left(-\tilde{q}_{-k}\right)\right. \\
& \left.-H_{n}\left(\tilde{q}_{-k}\right) H_{n}\left(-q_{k}\right) H_{m}\left(-q_{k}\right) H_{m}\left(\tilde{q}_{-k}\right)+H_{n}\left(-\tilde{q}_{-k}\right) H_{n}\left(-q_{k}\right) H_{m}\left(-q_{k}\right) H_{m}\left(-\tilde{q}_{-k}\right)\right] e^{-\tilde{q}_{k}^{2}-q_{k}^{2}} \text {, }
\end{aligned}
$$

where we recall that $H_{n}($.$) is a Hermite polynomial of order n$. This expression is made of four terms that can be calculated separately. Using the relation

$$
\sum_{n=0}^{+\infty} \frac{w^{n}}{n !} H_{n}(x) H_{n}(y)=\frac{1}{\sqrt{1-4 w^{2}}} \exp \left\{\frac{2 w}{4 w^{2}-1}\left[2 w\left(x^{2}+y^{2}\right)-2 x y\right]\right\}
$$

the first term reads

$$
\begin{aligned}
\left\langle\Psi_{2, \mathrm{sq}}\left|\hat{\mathcal{S}}_{x}(\boldsymbol{k}) \hat{\mathcal{S}}_{x}(-\boldsymbol{k})\right| \Psi_{2, \mathrm{sq}}\right\rangle^{(1)}= & \frac{1}{\pi \cosh ^{2} r_{k}} \frac{1}{\sqrt{1-4 w^{2}}} \frac{1}{\sqrt{1-4 w^{* 2}}} \int_{0}^{+\infty} \int_{0}^{+\infty} \mathrm{d} \tilde{q}_{-\boldsymbol{k}} \mathrm{d} q_{\boldsymbol{k}} \\
& \times \exp \left\{-q_{\boldsymbol{k}}^{2}+\frac{2 w}{4 w^{2}-1}\left[2 w\left(q_{\boldsymbol{k}}^{2}+\tilde{q}_{-\boldsymbol{k}}^{2}\right)-2 q_{\boldsymbol{k}} \tilde{q}_{-\boldsymbol{k}}\right]\right\} \\
& \times \exp \left\{-\tilde{q}_{\boldsymbol{k}}^{2}+\frac{2 w^{*}}{4 w^{* 2}-1}\left[2 w^{*}\left(q_{\boldsymbol{k}}^{2}+\tilde{q}_{-\boldsymbol{k}}^{2}\right)-2 q_{\boldsymbol{k}} \tilde{q}_{-\boldsymbol{k}}\right]\right\},
\end{aligned}
$$


where we have defined $w \equiv e^{-2 i \varphi_{k}} \tanh r_{k} / 2$. We see that the only thing which remains to be calculated is a two-dimensional Gaussian integral. Simplifying the argument of the exponential, it takes the following form

$$
\left\langle\Psi_{2, \mathrm{sq}}\left|\hat{\mathcal{S}}_{x}(\boldsymbol{k}) \hat{\mathcal{S}}_{x}(-\boldsymbol{k})\right| \Psi_{2, \mathrm{sq}}\right\rangle^{(1)}=\frac{1}{\pi \cosh ^{2} r_{k}} \frac{1}{\sqrt{1-4 w^{2}}} \frac{1}{\sqrt{1-4 w^{* 2}}} \int_{0}^{+\infty} \int_{0}^{+\infty} \mathrm{d} \tilde{q}_{-k} \mathrm{~d} q_{\boldsymbol{k}} e^{-A\left(\tilde{q}_{-k}^{2}+q_{k}^{2}\right)+C \tilde{q}_{-k} q_{k}}
$$

where the coefficients $A$ and $C$ can be expressed as

$$
\begin{aligned}
& A \equiv \frac{\tanh ^{2}\left(r_{k}\right)+1}{\cosh ^{2}\left(r_{k}\right)\left[\tanh ^{4}\left(r_{k}\right)-2 \tanh ^{2}\left(r_{k}\right) \cos \left(4 \varphi_{k}\right)+1\right]}, \\
& C \equiv \frac{4 \tanh \left(r_{k}\right) \cos \left(2 \varphi_{k}\right)}{\cosh ^{2}\left(r_{k}\right)\left[\tanh ^{4}\left(r_{k}\right)-2 \tanh ^{2}\left(r_{k}\right) \cos \left(4 \varphi_{k}\right)+1\right]} .
\end{aligned}
$$

The three other terms can also be calculated explicitly by using the remark that $H_{n}(-x)=(-1)^{n} H_{n}(x)$. Then, one immediately sees that the fourth term is in fact equal to the first one. It is also clear that the second and the third ones are equal. The calculation of these two last terms is very similar to the above calculation except that the quantity $w$ should be substituted by $-w$ which leads to an expression similar to Eq. (A8), the only difference being that the sign of the term $C \tilde{q}_{-k} q_{k}$ in the argument of the exponential is changed. The final result reads

$$
\begin{aligned}
\left\langle\Psi_{2, \mathrm{sq}}\left|\hat{\mathcal{S}}_{x}(\boldsymbol{k}) \hat{\mathcal{S}}_{x}(-\boldsymbol{k})\right| \Psi_{2, \mathrm{sq}}\right\rangle= & \frac{2}{\pi \cosh ^{2} r_{k}} \frac{1}{\sqrt{1-4 w^{2}}} \frac{1}{\sqrt{1-4 w^{* 2}}} \\
& \times \int_{0}^{+\infty} \int_{0}^{+\infty} \mathrm{d} \tilde{q}_{-k} \mathrm{~d} q_{\boldsymbol{k}}\left[e^{-A\left(\tilde{q}_{-k}^{2}+q_{k}^{2}\right)+C \tilde{q}_{-k} q_{k}}-e^{-A\left(\tilde{q}_{-k}^{2}+q_{k}^{2}\right)-C \tilde{q}_{-k} q_{k}}\right] .
\end{aligned}
$$

The integration can be performed explicitly and one obtains

$$
\left\langle\Psi_{2, \mathrm{sq}}\left|\hat{\mathcal{S}}_{x}(\boldsymbol{k}) \hat{\mathcal{S}}_{x}(-\boldsymbol{k})\right| \Psi_{2, \mathrm{sq}}\right\rangle=\frac{4}{\pi \cosh ^{2} r_{k}} \frac{1}{\sqrt{1-4 w^{2}}} \frac{1}{\sqrt{1-4 w^{* 2}}} \frac{1}{\sqrt{4 A^{2}-C^{2}}} \arctan \left(\frac{C}{\sqrt{4 A^{2}-C^{2}}}\right) .
$$

Finally, using the expression of $w$, one arrives at our final expression, namely

$$
\left\langle\Psi_{2, \mathrm{sq}}\left|\hat{\mathcal{S}}_{x}(\boldsymbol{k}) \hat{\mathcal{S}}_{x}(-\boldsymbol{k})\right| \Psi_{2, \mathrm{sq}}\right\rangle=\frac{2}{\pi} \arctan \left[\frac{2 \tanh \left(r_{k}\right) \cos \left(2 \varphi_{k}\right)}{\sqrt{\tanh ^{4}\left(r_{k}\right)-2 \tanh ^{2}\left(r_{k}\right) \cos \left(4 \varphi_{k}\right)+1}}\right] .
$$

This is the expression used in the main text. Let us also notice that, if the squeezing angle vanishes, $\varphi_{k}=0$, then one simply has

$$
\left\langle\Psi_{2, \mathrm{sq}}\left|\hat{\mathcal{S}}_{x}(\boldsymbol{k}) \hat{\mathcal{S}}_{x}(-\boldsymbol{k})\right| \Psi_{2, \mathrm{sq}}\right\rangle=\frac{2}{\pi} \arctan \left[\sinh \left(2 r_{k}\right)\right] .
$$

This expression is consistent with Eq. (35) of Ref. [52], except that, in that reference, there is probably a misprint in the expression of the Bell operator mean value which should rather look like Eq. (25) of the same article.

[1] A. A. Starobinsky, Phys. Lett. 91B, 99 (1980).

[2] K. Sato, Mon. Not. R. Astron. Soc. 195, 467 (1981).

[3] A. H. Guth, Phys. Rev. D 23, 347 (1981).

[4] A. D. Linde, Phys. Lett. 108B, 389 (1982).

[5] A. Albrecht and P. J. Steinhardt, Phys. Rev. Lett. 48, 1220 (1982).

[6] A. D. Linde, Phys. Lett. 129B, 177 (1983).
[7] P. Ade et al. (Planck Collaboration), Astron. Astrophys. 571, A16 (2014).

[8] P. Ade et al. (Planck Collaboration), Astron. Astrophys. 571, A22 (2014).

[9] P. Ade et al. (Planck Collaboration), Astron. Astrophys. 571, A24 (2014).

[10] P. A. R. Ade et al. (Planck Collaboration), Astron. Astrophys. 594, A13 (2016). 
[11] P. A. R. Ade et al. (Planck Collaboration), Astron. Astrophys. 594, A20 (2016).

[12] P. A. R. Ade et al. (Planck Collaboration), Astron. Astrophys. 594, A17 (2016).

[13] V. F. Mukhanov and G. Chibisov, JETP Lett. 33, 532 (1981).

[14] V. F. Mukhanov and G. Chibisov, Sov. Phys. JETP 56, 258 (1982).

[15] A. A. Starobinsky, Phys. Lett. 117B, 175 (1982).

[16] A. H. Guth and S. Pi, Phys. Rev. Lett. 49, 1110 (1982).

[17] S. Hawking, Phys. Lett. 115B, 295 (1982).

[18] J. M. Bardeen, P. J. Steinhardt, and M. S. Turner, Phys. Rev. D 28, 679 (1983).

[19] V. F. Mukhanov, H. Feldman, and R. H. Brandenberger, Phys. Rep. 215, 203 (1992).

[20] J. Martin, Braz. J. Phys. 34, 1307 (2004).

[21] J. Martin, Lect. Notes Phys. 669, 199 (2005).

[22] J. Martin, Lect. Notes Phys. 738, 193 (2008).

[23] A. D. Linde, Lect. Notes Phys. 738, 1 (2008).

[24] L. Sriramkumar, arXiv:0904.4584.

[25] V. Vennin, J. Martin, and C. Ringeval, C.R. Phys. 16, 960 (2015).

[26] L. Grishchuk and Y. Sidorov, Phys. Rev. D 42, 3413 (1990).

[27] L. Grishchuk, H. Haus, and K. Bergman, Phys. Rev. D 46, 1440 (1992).

[28] D. Polarski and A. A. Starobinsky, Classical Quantum Gravity 13, 377 (1996).

[29] J. Martin and V. Vennin, Phys. Rev. D 93, 023505 (2016).

[30] D. Campo and R. Parentani, Phys. Rev. D 74, 025001 (2006).

[31] J. Maldacena, Fortschr. Phys. 64, 10 (2016).

[32] J. Martin and V. Vennin, Phys. Rev. A 93, 062117 (2016).

[33] S. Choudhury, S. Panda, and R. Singh, Eur. Phys. J. C 77, 60 (2017).

[34] J.-W. Chen, S.-H. Dai, D. Maity, S. Sun, and Y.-L. Zhang, arXiv:1701.03437.

[35] S. Kanno and J. Soda, arXiv:1705.06199.

[36] S. M. Leach, M. Sasaki, D. Wands, and A. R. Liddle, Phys. Rev. D 64, 023512 (2001).

[37] L. Henderson and V. Vedral, J. Phys. A 34, 6899 (2001).

[38] H. Ollivier and W. H. Zurek, Phys. Rev. Lett. 88, 017901 (2001).

[39] G. Adesso, T. R. Bromley, and M. Cianciaruso, J. Phys. A 49, 473001 (2016).

[40] R. F. Werner, Phys. Rev. A 40, 4277 (1989).

[41] G. Adesso and A. Datta, Phys. Rev. Lett. 105, 030501 (2010).

[42] S. Rahimi-Keshari, C. M. Caves, and T. C. Ralph, Phys. Rev. A 87, 012119 (2013).

[43] L. Mišta, D. McNulty, and G. Adesso, Phys. Rev. A 90, 022328 (2014).

[44] J. F. Clauser, M. A. Horne, A. Shimony, and R. A. Holt, Phys. Rev. Lett. 23, 880 (1969).

[45] A. Aspect, J. Dalibard, and G. Roger, Phys. Rev. Lett. 49, 1804 (1982).

[46] A. Aspect, P. Grangier, and G. Roger, Phys. Rev. Lett. 49, 91 (1982).
[47] G. Weihs, T. Jennewein, C. Simon, H. Weinfurter, and A. Zeilinger, Phys. Rev. Lett. 81, 5039 (1998).

[48] B. Hensen et al., Nature (London) 526, 682 (2015).

[49] J. Handsteiner et al., Phys. Rev. Lett. 118, 060401 (2017).

[50] K. Banaszek and K. Wódkiewicz, Phys. Rev. Lett. 82, 2009 (1999).

[51] Z.-B. Chen, J.-W. Pan, G. Hou, and Y.-D. Zhang, Phys. Rev. Lett. 88, 040406 (2002).

[52] M. Revzen, Found. Phys. 36, 546 (2006).

[53] M. Revzen, P. A. Mello, A. Mann, and L. M. Johansen, Phys. Rev. A 71, 022103 (2005).

[54] B. S. Cirel'Son, Lett. Math. Phys. 4, 93 (1980).

[55] A. Einstein, B. Podolsky, and N. Rosen, Phys. Rev. 47, 777 (1935).

[56] G. Gour, F. C. Khanna, A. Mann, and M. Revzen, Phys. Lett. A 324, 415 (2004).

[57] I. S. Gradshteyn and I. M. Ryzhik, Table of Integrals, Series, and Products (Academic Press, New York, 1965).

[58] J.-Å. Larsson, Phys. Rev. A 70, 022102 (2004).

[59] A. Perez, H. Sahlmann, and D. Sudarsky, Classical Quantum Gravity 23, 2317 (2006).

[60] D. Sudarsky, Int. J. Mod. Phys. D 20, 509 (2011).

[61] A. Bassi, K. Lochan, S. Satin, T. P. Singh, and H. Ulbricht, Rev. Mod. Phys. 85, 471 (2013).

[62] J. Martin, V. Vennin, and P. Peter, Phys. Rev. D 86, 103524 (2012).

[63] P. Caate, P. Pearle, and D. Sudarsky, Phys. Rev. D 87, 104024 (2013).

[64] S. Das, K. Lochan, S. Sahu, and T. P. Singh, Phys. Rev. D 88, 085020 (2013); 89, 109902(E) (2014).

[65] S. Das, S. Sahu, S. Banerjee, and T. P. Singh, Phys. Rev. D 90, 043503 (2014).

[66] G. Len and G. R. Bengochea, Eur. Phys. J. C 76, 29 (2016).

[67] V. Mukhanov, in Universe or Multiverse, edited by Bernard Carr (Springer, Berlin, 2007), p. 267.

[68] C. Kiefer and D. Polarski, Adv. Sci. Lett. 2, 164 (2009).

[69] A. Valentini, Phys. Rev. D 82, 063513 (2010).

[70] N. Pinto-Neto, G. Santos, and W. Struyve, Phys. Rev. D 85, 083506 (2012).

[71] S. Goldstein, W. Struyve, and R. Tumulka, arXiv:1508.01017.

[72] S. Colin and A. Valentini, Int. J. Mod. Phys. D 25, 1650068 (2016).

[73] W. H. Zurek, Phys. Rev. D 24, 1516 (1981).

[74] W. H. Zurek, Phys. Rev. D 26, 1862 (1982).

[75] E. Joos and H. D. Zeh, Z. Phys. B 59, 223 (1985).

[76] J. P. Paz and W. H. Zurek, Phys. Rev. D 48, 2728 (1993).

[77] E. Calzetta and B. L. Hu, Phys. Rev. D 52, 6770 (1995).

[78] M. Franco and E. Calzetta, Classical Quantum Gravity 28, 145024 (2011).

[79] D. Giulini, C. Kiefer, E. Joos, J. Kupsch, I. O. Stamatescu, and H.D. Zeh, Decoherence and the Appearance of a Classical World in Quantum Theory (Cambridge University Press, Cambridge, 1996).

[80] A. J. Leggett and A. Garg, Phys. Rev. Lett. 54, 857 (1985).

[81] C. Emary, N. Lambert, and F. Nori, Rep. Prog. Phys. 77, 016001 (2014).

[82] J. Martin and V. Vennin, Phys. Rev. A 94, 052135 (2016). 